\title{
Sensing-Throughput Tradeoff for Interweave Cognitive Radio System: A Deployment-Centric Viewpoint
}

\author{
Ankit Kaushik $^{*}$, Student Member, IEEE, Shree Krishna Sharma ${ }^{\dagger}$, Member, IEEE, \\ Symeon Chatzinotas ${ }^{\dagger}$, Senior Member, IEEE, Björn Ottersten ${ }^{\dagger}$, Fellow, IEEE, \\ Friedrich K. Jondral ${ }^{*}$ Senior Member, IEEE
}

\begin{abstract}
Secondary access to the licensed spectrum is viable only if the interference is avoided at the primary system. In this regard, different paradigms have been conceptualized in the existing literature. Among these, interweave systems (ISs) that employ spectrum sensing have been widely investigated. Baseline models investigated in the literature characterize the performance of the IS in terms of a sensing-throughput tradeoff, however, this characterization assumes perfect knowledge of the involved channels at the secondary transmitter, which is unavailable in practice. Motivated by this fact, we establish a novel approach that incorporates channel estimation in the system model, and consequently investigate the impact of imperfect channel knowledge on the performance of the IS. More particularly, the variation induced in the detection probability affects the detector's performance at the secondary transmitter, which may result in severe interference at the primary receivers. In this view, we propose to employ average and outage constraints on the detection probability, in order to capture the performance of the IS. Our analysis reveals that with an appropriate choice of the estimation time determined by the proposed approach, the performance degradation of the IS can be effectively controlled, and subsequently the achievable secondary throughput can be significantly enhanced.
\end{abstract}

Index Terms-Cognitive radio, Interweave system, Sensingthroughput tradeoff, Spectrum Sensing, Channel estimation

\section{INTRODUCTION}

We are currently in the phase of conceptualizing the requirements of the fifth generation $(5 \mathrm{G})$ of mobile wireless systems. One of the major goals is to improve the areal capacity (bits $/ \mathrm{s} / \mathrm{m}^{2}$ ) by a factor of 1000 [2]. To this end, an extension to the already allocated spectrum is of paramount importance. Recently, the spectrum beyond $6 \mathrm{GHz}$, which largely entails the millimeter wave is envisaged as a powerful source of spectrum for $5 \mathrm{G}$ wireless systems. However, the millimeter wave technology is still in its initial stage and along with complex regulatory requirements in this regime, it has to address several challenges like propagation loss, low efficiency

\footnotetext{
${ }^{*}$ A. Kaushik and F. K. Jondral are with Communications Engineering Lab, Karlsruhe Institute of Technology (KIT), Germany. Email:\{ankit.kaushik,friedrich.jondral\}@ kit.edu.

${ }^{\dagger}$ S.K. Sharma, S. Chatzinotas and B. Ottersten are with SnT securityandtrust.lu, University of Luxembourg, Luxembourg. Email:\{shree.sharma, symeon.chatzinotas, bjorn.ottersten\}@uni.lu.

The preliminary analysis of this paper has been presented at CROWNCOM 2015 in Doha, Qatar [1].

This work was partially supported by the National Research Fund, Luxembourg under the CORE projects "SeMIGod" and "SATSENT".
}

of radio frequency components such as power amplifiers, small size of the antenna and link acquisition [3]. Therefore, in order to capture a deeper insight of its feasibility in 5G, it is essential to overcome the aforementioned challenges in the near future.

Besides the spectrum beyond $6 \mathrm{GHz}$, an efficient utilization of the spectrum below $6 \mathrm{GHz}$ presents an alternative solution. The use of the spectrum in this regime (below $6 \mathrm{GHz}$ ) is fragmented and statically allocated, leading to inefficiencies and the shortage in the availability of spectrum for new services. However, it is possible to overcome this scarcity if we manage to utilize this radio spectrum efficiently. In this perspective, cognitive radio (CR) is foreseen as one of the potential contenders that addresses the spectrum scarcity problem. Since its origin by Mitola et al. in 1999, this notion has evolved at a significant pace, and consequently has acquired certain maturity. However, from a deployment perspective, this technology is still in its preliminary phase. In this view, it is necessary to make substantial efforts that enable the placement of this concept over a hardware platform.

An access to the licensed spectrum is an outcome to the paradigm employed by the secondary user (SU). Based on the paradigms described in the literature, all CR systems that provide dynamic access to the spectrum mainly fall under three categories, namely, interweave, underlay and overlay systems [4]. In interweave systems (ISs), the SUs render an interference-free access to the licensed spectrum by exploiting spectral holes in different domains such as time, frequency, space and polarization, whereas underlay systems enable an interference-tolerant access under which the SUs are allowed to use the licensed spectrum (e.g. Ultra Wide Band) as long as they respect the interference constraints of the primary receivers (PRs). Besides that, overlay systems consider the participation of higher layers for enabling the spectral coexistence between two or more wireless networks. Due to its ease of deployment, the IS is mostly preferred not only for performing theoretical analysis but also for practical implementation as well. Motivated by these facts, this paper focuses on the performance analysis of the ISs from a deployment perspective.

\section{A. Motivation and Related Work}

Spectrum sensing is an integral part of ISs. At the secondary transmitter (ST), sensing is necessary for detecting the presence or the absence of a primary user (PU) signal, thereby 
protecting the PRs against harmful interference. A sensing mechanism at the ST can be accomplished by listening to the signal transmitted by the primary transmitter (PT). For detecting a PU signal, several techniques such as energy detection, matched filtering, cyclostationary and feature-based detection exist [5], [6]. Because of its versatility towards unknown PU signals and its low computational complexity, energy detection has been extensively investigated in the literature [7]-[11]. In this technique, the decision is accomplished by comparing the power received at the ST to a decision threshold. In reality, the ST encounters variations in the received power due to the existence of thermal noise at the receiver and channel fading. Subsequently, these variations lead to sensing errors described as misdetection or false alarm, which limit the performance of the IS. In order to determine the performance of a detector, it is essential to obtain the expressions of detection probability and false alarm probability.

In particular, detection probability is critical for ISs because it protects the PR from the interference induced by the ST. As a result, the ISs have to ensure that they operate above a target detection probability [12]. Therefore, the characterization of the detection probability becomes absolutely necessary for the performance analysis of the IS. In this context, Urkowitz [7] introduced a probabilistic framework for characterizing the sensing errors, however, the characterization accounts only for the noise in the system. To encounter the variation caused by channel fading, a frame structure has been introduced in [13] assuming that the channel remains constant over the frame duration, however, upon exceeding the frame duration, the system may observe a different realization of the channel. Based on this frame structure, the performance of the IS has been investigated in terms of deterministic channel [13]-[15] and random channel ${ }^{1}[8]-[10]$. Complementing the analysis in [13]-[15], in this paper, we consider the involved channels to be deterministic.

Besides the detection probability, false alarm probability has a large influence on the achievable throughput of the secondary system. Recently, the performance characterization of CR systems in terms of a sensing-throughput tradeoff has received significant attention [13], [15]-[17]. According to Liang et al. [13], the ST assures a reliable detection of a PU signal by retaining the detection probability above a desired level with an objective of maximizing the throughput at the secondary receiver (SR). In this way, the sensing-throughput tradeoff depicts a suitable sensing time that achieves a maximum secondary throughput. However, to characterize the detection probability and the secondary throughput, the system requires the knowledge of interacting channels, namely, a sensing channel, an access channel and an interference channel, refer to Fig. $1^{2}$. To the best of authors' knowledge, the baseline models investigated in the literature assume the knowledge of these channels to be available at the ST. However, in practice, this knowledge is not available, thus, needs to be estimated

\footnotetext{
${ }^{1}$ In the literature, deterministic and random channels are interpreted as pathloss and fading channels, respectively.

${ }^{2}$ As the interference to the PR is controlled by a regulatory constraint over the detection probability, in this view, the interaction with the PR is excluded in the considered scenario [13]
}

by the secondary system. As a result, from a deployment perspective, the existing solutions for the IS are considered inaccurate for the performance analysis.

In practice, the knowledge about the involved channels can be estimated either (i) directly by using the conventional channel estimation techniques such as training sequence based [18] and pilot based [19], [20] channel estimation or (ii) indirectly by estimating the received signal to noise ratio [21], [22]. It is worthy to note that the sensing and interference channels represent the channels between two different (primary and secondary) systems. In this context, it becomes challenging to select the estimation methods in such a way that low complexity and versatility (towards different PU signals) requirements are satisfied. These issues, discussed later in Section III-B, render the existing estimation techniques [18][22] unsuitable for hardware implementations. To this end, we propose to employ a received power based estimation at the ST and at the SR for the sensing and interference channels, respectively. Considering the fact that the access channel corresponds to the link between the ST and the SR, we propose to employ conventional channel estimation techniques such as pilot based channel estimation at the SR.

Inherent to the estimation process, the variations due the channel estimation translate to variations in the performance parameters, namely detection probability and secondary throughput. In particular, the variations induced in the detection probability may result in harmful interference at the PR, hence, severely degrading the performance of a CR system. In this context, the performance characterization of an IS with imperfect channel knowledge remains an open problem. In this regard, this paper focuses on the performance characterization of the IS in terms of sensing-throughput tradeoff taking these aforementioned aspects into account.

\section{B. Contributions}

The major contributions of this paper can be summarized as follows:

1) Analytical framework: In contrast to the existing models that assume the perfect knowledge of the channels, the main goal of this paper is to derive an analytical framework that constitutes the estimation of: (i) sensing channel at the ST, (ii) access channel and (iii) interference channel at the SR. Under this framework, we propose a novel integration of the channel estimation in the secondary system's frame structure, according to which, we take into account the samples considered for channel estimation (of the sensing channel) also for sensing in such a way that the time resources within the frame are utilized efficiently. Furthermore, we select the estimation techniques in such a way that the hardware complexity and the versatility towards unknown PU signals requirements (as considered while employing an energy based detection) are not compromised. In this context, we propose to employ a received power based estimation for the sensing and interference channels. Based on this framework, we characterize the performance of the IS by considering: (i) the variations due to imperfect channel knowledge and (ii) the performance degradation due to the inclusion of channel estimation. 
2) Imperfect channel knowledge: To capture the variations induced due to imperfect channel knowledge, we characterize the distribution functions of performance parameters such as detection probability and achievable secondary throughput. More importantly, we utilize the distribution function of the detection probability to incorporate two primary user (PU) constraints, namely, average and outage constraints on the detection probability. In this way, the proposed approach is able to control the amount of excessive interference caused at the PR due to the imperfect channel knowledge.

3) Estimation-sensing-throughput tradeoff: Subject to the average and the outage constraints, we establish the expressions of sensing-throughput tradeoff that capture the aforementioned variations and evaluate the performance loss in terms of the achievable secondary throughput. In particular, we propose two different optimization approaches for countering the variations in sensing-throughput tradeoff and determining a suitable sensing time, which attains a maximum secondary throughput. Finally, we depict a fundamental tradeoff between estimation time, sensing time and achievable secondary throughput. We exploit this tradeoff to determine a suitable estimation and sensing time that depicts the maximum achievable performance of the IS.

\section{Organization}

The subsequent sections of the paper are organized as follows: Section II describes the system model that includes the deployment scenario and the signal model. Section III presents the problem description and the proposed approach. Section IV characterizes the distribution functions of the performance parameters and establishes the sensing-throughput tradeoff subject to average and outage constraints. Section V analyzes the numerical results based on the obtained expressions. Finally, Section VI concludes the paper. Table I lists the definitions of acronyms and important mathematical notations used throughput the paper.

\section{SYSTEM MODEL}

\section{A. Deployment Scenario}

The cognitive small cell (CSC), a CR application, characterizes a small cell deployment that fulfills the spectral requirements for mobile stations (MSs) operating indoor, refer to Fig. 1. For the disposition of the CSC in the network, the following key elements are essential: a CSC-base station (CSC-BS), a macro cell-base station (MC-BS) and MS, refer to Fig. 1. MSs are the indoor devices served by the CSC-BS over an access channel $\left(h_{\mathrm{s}}\right)$. Furthermore, the MC-BS is connected to several CSC-BSs over a wireless backhaul ${ }^{3}$. Moreover, the transmissions from the PT can be listened by the CSC-BS and the MS over sensing $\left(h_{\mathrm{p}, 1}\right)$ and interference channel $\left(h_{\mathrm{p}, 2}\right)$, respectively. Considering the fact that the IS is employed at the CSC-BS, the CSC-BS and the MS represent ST and SR, respectively. A hardware prototype of the CSC-BS operating

${ }^{3} \mathrm{~A}$ wireless backhaul is a point-to-point wireless link between the CSCBS and MC-BS that relays the traffic generated from the CSC to the core network.
TABLE I

DEFINITIONS OF ACRONYMS AND NOTATIONS USED

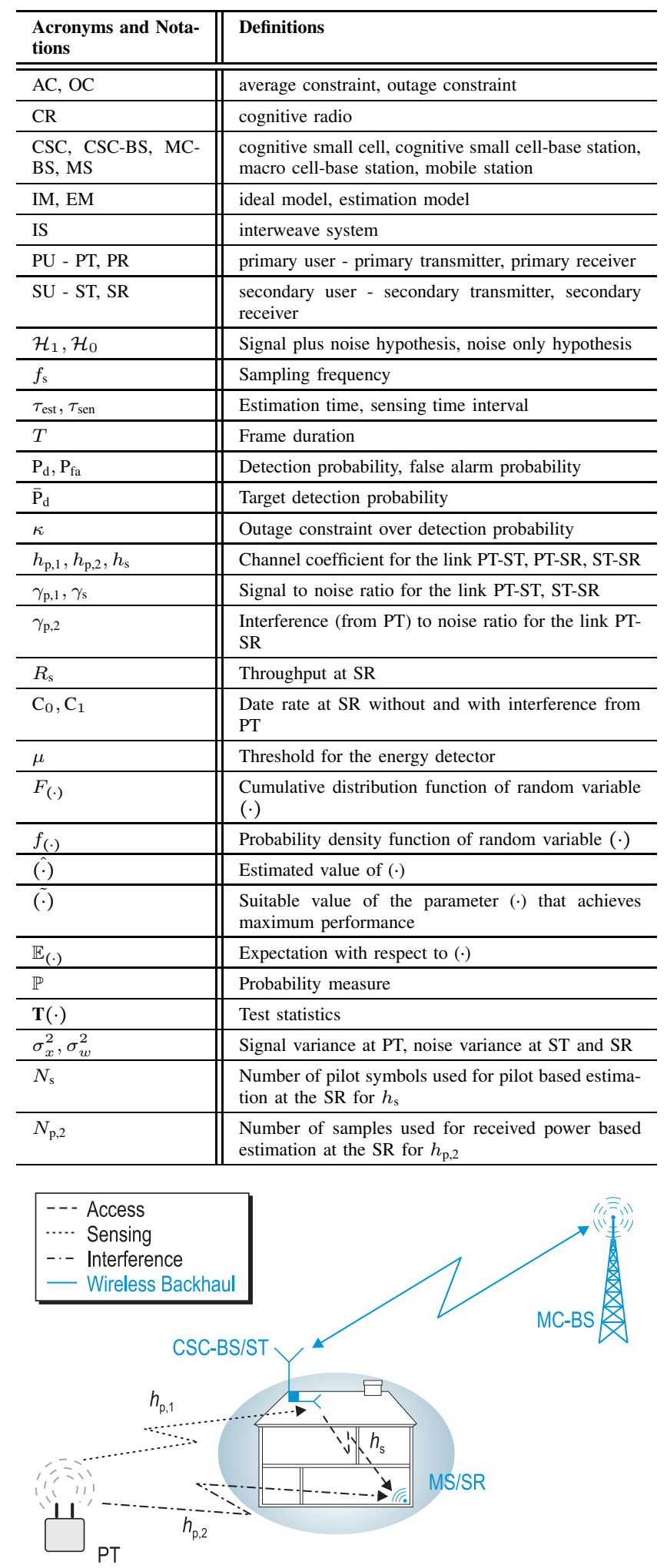

Fig. 1. A cognitive small cell scenario demonstrating: (i) the interweave paradigm, (ii) the associated network elements, which constitute cognitive small cell-base station/secondary transmitter (CSC-BS/ST), mobile station/secondary receiver (MS/SR), macro cell-base station (MC-BS) and primary transmitter (PT), (iii) the interacting channels: sensing $\left(h_{\mathrm{p}, 1}\right)$, access $\left(h_{\mathrm{s}}\right)$ and interference $\left(h_{\mathrm{p}, 2}\right)$. 
as IS was presented in [23]. For simplification, a PU constraint based on false alarm probability was considered in [23]. With the purpose of improving system's reliability, we extend the analysis to employ a PU constraint on the detection probability.

Complementing the analysis depicted in [13], we consider a slotted medium access for the IS, where the time axis is segmented into frames of length $T$, according to which, the ST employs periodic sensing. Hence, each frame consists of a sensing slot $\tau_{\text {sen }}$ and the remaining duration $T-\tau_{\text {sen }}$ is utilized for data transmission. For small $T$ relative to the PUs' expected ON/OFF period, the requirement of the ST to be in alignment to PUs' medium access can be relaxed [24]-[26].

\section{B. Signal model}

Subject to the underlying hypothesis that illustrates the presence $\left(\mathcal{H}_{1}\right)$ or absence $\left(\mathcal{H}_{0}\right)$ of a PU signal, the discrete and real signal received at the ST is given by

$$
y_{\mathrm{ST}}[n]=\left\{\begin{array}{ll}
h_{\mathrm{p}, 1} \cdot x_{\mathrm{PT}}[n]+w[n] & : \mathcal{H}_{1} \\
w[n] & : \mathcal{H}_{0}
\end{array},\right.
$$

where $x_{\mathrm{PT}}[n]$ corresponds to a discrete and real sample transmitted by the PT, $\left|h_{\mathrm{p}, 1}\right|^{2}$ represents the power gain of the sensing channel for a given frame and $w[n]$ is additive white Gaussian noise at the ST. According to [13], the signal $x_{\mathrm{PT}}[n]$ transmitted by the PUs can be modelled as: (i) phase shift keying modulated signal, or (ii) Gaussian signal. The signals that are prone to high inter-symbol interference or entail precoding can be modelled as Gaussian signals. For this paper, we focus our analysis on the latter case. As a result, the mean and the variance for the signal and the noise are determined as $\mathbb{E}\left[x_{\mathrm{PT}}[n]\right]=0, \mathbb{E}[w[n]]=0$, $\mathbb{E}\left[\left|x_{\mathrm{PT}}[n]\right|^{2}\right]=\sigma_{x}^{2}$ and $\mathbb{E}\left[|w[n]|^{2}\right]=\sigma_{w}^{2}$. The channel $h_{\mathrm{p}, 1}$ is considered to be independent of $x_{\mathrm{PT}}[n]$ and $w[n]$, thus, $y_{\mathrm{ST}}$ is also an independent and identically distributed (i.i.d.) random process.

Similar to (1), during data transmission, the discrete and real received signal at the SR conditioned on the detection probability $\left(\mathrm{P}_{\mathrm{d}}\right)$ and false alarm probability $\left(\mathrm{P}_{\mathrm{fa}}\right)$ is given by

$$
y_{\mathrm{SR}}[n]=\left\{\begin{array}{ll}
h_{\mathrm{S}} \cdot x_{\mathrm{ST}}[n]+h_{\mathrm{p}, 2} \cdot x_{\mathrm{PT}}[n]+w[n] & : 1-\mathrm{P}_{\mathrm{d}} \\
h_{\mathrm{S}} \cdot x_{\mathrm{ST}}[n]+w[n] & : 1-\mathrm{P}_{\mathrm{fa}}
\end{array},\right.
$$

where $x_{\mathrm{ST}}[n]$ corresponds to discrete and real sample transmitted by the ST. Further, $\left|h_{\mathrm{s}}\right|^{2}$ and $\left|h_{\mathrm{p}, 2}\right|^{2}$ represent the power gains for the access and the interference channels, refer to Fig. 1.

\section{Problem Description And Proposed Approach}

\section{A. Problem Description}

In accordance with the conventional frame structure, the ST performs sensing for a duration of $\tau_{\text {sen }}$. The test statistics $\mathrm{T}(\mathbf{y})$ at the ST is evaluated as

$$
\mathrm{T}(\mathbf{y})=\frac{1}{\tau_{\text {sen }} f_{\mathrm{s}}} \sum_{n=1}^{\tau_{\text {sen }} f_{\mathrm{s}}}\left|y_{\mathrm{ST}}[n]\right|^{2} \underset{\mathcal{H}_{0}}{\stackrel{\mathcal{H}_{1}}{\gtrless}} \mu,
$$

where $\mu$ is the decision threshold and $\mathbf{y}$ is a vector with $\tau_{\text {sen }} f_{\mathrm{s}}$ samples. $\mathrm{T}(\mathbf{y})$ represents a random variable, whereby the characterization of the distribution function depends on the underlying hypothesis. Corresponding to $\mathcal{H}_{0}$ and $\mathcal{H}_{1}$, $\mathrm{T}(\mathbf{y})$ follows a central chi-squared $\left(\mathcal{X}^{2}\right)$ distribution [27]. As a result, the detection probability $\left(\mathrm{P}_{\mathrm{d}}\right)$ and the false alarm probability $\left(\mathrm{P}_{\mathrm{fa}}\right)$ corresponding to (3) are determined as [28]

$$
\begin{gathered}
\mathrm{P}_{\mathrm{d}}\left(\mu, \tau_{\mathrm{sen}}, P_{\mathrm{Rx}, \mathrm{ST}}\right)=\Gamma\left(\frac{\tau_{\mathrm{sen}} f_{\mathrm{s}}}{2}, \frac{\tau_{\mathrm{sen}} f_{\mathrm{s}} \mu}{2 P_{\mathrm{Rx}, \mathrm{ST}}}\right), \\
\mathrm{P}_{\mathrm{fa}}\left(\mu, \tau_{\mathrm{sen}}\right)=\Gamma\left(\frac{\tau_{\mathrm{sen}} f_{\mathrm{s}}}{2}, \frac{\tau_{\mathrm{sen}} f_{\mathrm{s}} \mu}{2 \sigma_{w}^{2}}\right),
\end{gathered}
$$

where $P_{\mathrm{Rx}, \mathrm{ST}}$ is the power received over the sensing channel and $\Gamma(\cdot, \cdot)$ represents a regularized incomplete upper Gamma function [29].

Following the characterization of $\mathrm{P}_{\mathrm{fa}}$ and $\mathrm{P}_{\mathrm{d}}$, Liang et al. [13] established a tradeoff between the sensing time and secondary throughput $\left(R_{\mathrm{s}}\right)$ subject to a target detection probability $\left(\overline{\mathrm{P}}_{\mathrm{d}}\right)$. This tradeoff is represented as

$$
\begin{aligned}
& R_{\mathrm{s}}\left(\tilde{\tau}_{\mathrm{sen}}\right)=\max _{\tau_{\mathrm{sen}}} R_{\mathrm{s}}\left(\tau_{\mathrm{sen}}\right)=\frac{T-\tau_{\mathrm{sen}}}{T}\left[\mathrm{C}_{0}\left(1-\mathrm{P}_{\mathrm{fa}}\right) \mathbb{P}\left(\mathcal{H}_{0}\right)+\right. \\
& \left.\quad \mathrm{C}_{1}\left(1-\mathrm{P}_{\mathrm{d}}\right) \mathbb{P}\left(\mathcal{H}_{1}\right)\right] \\
& \text { s.t. } \mathrm{P}_{\mathrm{d}} \geq \overline{\mathrm{P}}_{\mathrm{d}}, \\
& \text { where } \mathrm{C}_{0}=\log _{2}\left(1+\left|h_{\mathrm{s}}\right|^{2} \frac{P_{\mathrm{Tx}, \mathrm{ST}}}{\sigma_{w}^{2}}\right)=\log _{2}\left(1+\gamma_{\mathrm{s}}\right) \\
& \text { and } \mathrm{C}_{1}=\log _{2}\left(1+\frac{\left|h_{\mathrm{s}}\right|^{2} P_{\mathrm{Tx}, \mathrm{ST}}}{\left|h_{\mathrm{p}, 2}\right|^{2} P_{\mathrm{Tx}, \mathrm{PT}}+\sigma_{w}^{2}}\right) \\
& \quad=\log _{2}\left(1+\frac{\left|h_{\mathrm{s}}\right|^{2} P_{\mathrm{Tx}, \mathrm{ST}}}{P_{\mathrm{Rx}, \mathrm{SR}}}\right)=\log _{2}\left(1+\frac{\gamma_{\mathrm{s}}}{\gamma_{\mathrm{p}, 2}+1}\right)
\end{aligned}
$$

where $\mathbb{P}\left(\mathcal{H}_{0}\right)$ and $\mathbb{P}\left(\mathcal{H}_{1}\right)$ are the occurrence probabilities for the respective hypothesis, whereas $\gamma_{\mathrm{p}, 2}$ and $\gamma_{\mathrm{s}}$ correspond to interference (from the PT) to noise ratio and signal to noise ratio for the links PT-SR and ST-SR, respectively. Moreover, $P_{\mathrm{Tx}, \mathrm{ST}}$ and $P_{\mathrm{Tx}, \mathrm{PT}}$ represent the transmit power at the PT and the ST, whereas $P_{\mathrm{Rx}, \mathrm{SR}}$ corresponds to the received power (which includes interference power from the PT and the noise power) at the SR. In addition, $\mathrm{C}_{0}$ and $\mathrm{C}_{1}$ represent the data rate without and with interference from the PT. In other words, using (6), the ST determines a suitable sensing time $\tau_{\text {sen }}=\tilde{\tau}_{\text {sen }}$, such that the secondary throughput is maximized subject to a target detection probability, refer to (7). From the deployment perspective, the tradeoff depicted above has the following fundamental issues:

- Without the knowledge of the received power $P_{\mathrm{Rx}, \mathrm{ST}}$ over the sensing channel, it is not feasible to characterize $P_{d}$, refer to (4). This leaves the characterization of the throughput (6) impossible and the constraint defined in (7) inappropriate.

- Moreover, the knowledge of the interference and the access channels is required at the ST, refer to (8) and (9) for characterizing the throughput in terms of $C_{0}$ and $\mathrm{C}_{1}$ at the SR.

Taking these issues into account, it is not feasible to employ the performance analysis depicted by this model (referred as 
ideal model, hereafter) for hardware implementation. In the subsequent section, we propose an analytical framework (also referred as estimation model) that addresses the aforementioned issues, thereby including the estimation of the sensing channel at the ST, and the interference and the access channels at the SR. Based on the proposed approach, we then investigate the performance of the IS in terms of the sensing-throughput tradeoff.

\section{B. Proposed Approach}

In order to overcome the difficulties discussed in Section III-A, the following strategy is proposed in this paper.

1) As a first step, we consider the estimation of the involved channels. In order to characterize the detection probability, we propose to employ a received power based estimation at the ST for the sensing channel. This is done to ensure that detection probability remains above a desired level. We further to employ a pilot based estimation and a received power based estimation for the access channel and the interference channel, respectively, at the $\mathrm{SR}$, to characterize the secondary throughput.

2) Next, we characterize the variations due to channel estimation in the estimated parameters, namely, received power (for the sensing and the interference channels) and the power gain (for the access channel) in terms of their cumulative distribution functions.

3) In order to investigate the performance of the IS subject to the channel estimation, we further characterize these variations in the performance parameters, which include detection probability and secondary throughput, in terms of their cumulative distribution functions.

4) Finally, we utilize the derived cumulative distribution functions to obtain the expressions of sensingthroughput tradeoff. Hence, based on these expressions, we quantify the impact of imperfect channel knowledge on performance of the ISs, and subsequently determine the achievable secondary throughput at a suitable sensing time.

It is well-known that systems with transmitter information (which includes the filter parameters, pilot symbols, modulation type and time-frequency synchronization) at the receiver acquire channel knowledge by listening to the pilot data sent by the ST [19], [20], [30], [31]. Other systems, where the receiver possesses either no access to this information or limited by hardware complexity, procure channel knowledge indirectly by estimating a different parameter that entails the channel knowledge, for instance, received signal power [1] or received signal to noise ratio [21], [22]. Recently, estimation techniques such as pilot based estimation [32], [33] and received power based estimation [34] have been applied to obtain channel knowledge for CR systems. However, the performance analysis has been limited to underlay systems, where the emphasis has been given on modelling the interference at the PR.

Since the pilot based estimation requires the knowledge of the PU signal at the secondary system, the versatility (in terms of PU signals) of the secondary system is compromised. On the other side, for the estimation of the received signal to noise ratio, Eigenvalue (which involves matrix operations) based approach [22] or iterative approaches such as expectationmaximization have been proposed [21]. Due to the complicated mathematical operations or the complexity of the iterative algorithms, such approaches tend to increase the hardware complexity of the ISs. In order to resolve these issues, we propose to employ received power based estimation for the sensing and interference channels, and pilot based estimation for the access channel. Similar to the energy based detection, since the received power based estimation involves simple operations on the obtained samples such as magnitude squared followed by summation, the proposed estimation provides a reasonable tradoff between complexity and versatility.

However, with the inclusion of this estimation, the system anticipates: (i) a performance loss in terms of temporal resources used and (ii) variations in the aforementioned performance parameters due to estimation. A preliminary analysis of this performance loss was carried out in [1], where it was revealed that in low signal to noise ratio regime, imperfect knowledge of received power corresponds to large variation in detection probability, hence, causes a severe degradation in the performance of the IS. However, this performance degradation was determined by means of lower and upper bounds. In this work, we consider a more exact analysis, whereby we capture the variations in detection probability by characterizing its distribution function, and subsequently apply new probabilistic constraints on the detection probability, which allow ISs to operate at low signal to noise ratio regime.

In order to include channel estimation, we propose a frame structure that constitutes an estimation $\tau_{\text {est }}$, a sensing $\tau_{\text {sen }}$ and data transmission $T-\tau_{\text {sen }}$, where $\tau_{\text {est }}$ and $\tau_{\text {sen }}$ correspond to time intervals and $0<\tau_{\text {est }} \leq \tau_{\text {sen }}<T$, refer to Fig. 2. Since the estimated values of the interacting channels are required for determining the suitable sensing time (the duration of the sensing phase), the sequence depicted in Fig. 2, whereby estimation followed by sensing is reasonable for the hardware deployment. Particularly for the sensing channel, it is worthy to note that the samples used for estimation can be combined with the samples acquired for sensing ${ }^{4}$ such that the time resources within the frame duration can be utilized efficiently, as shown in the frame structure in Fig. 2. To avail the estimates for the interference and access channels at the ST, a low-rate feedback channel from the SR to the ST is required for the proposed approach. In the following paragraphs, we consider the estimation of the involved channels.

1) Estimation of sensing channel $\left(h_{p, 1}\right)$ : Following the previous discussions, the ST acquires the knowledge of $h_{\mathrm{p}, 1}$ by estimating its received power. The estimated received power is required for the characterization of $\mathrm{P}_{\mathrm{d}}$, thereby evaluating the detector performance.

Under $\mathcal{H}_{1}$, the received power based estimated during the estimation phase at the ST is given as [7]

$$
\hat{P}_{\mathrm{Rx}, \mathrm{ST}}=\frac{1}{\tau_{\text {est }} f_{\mathrm{s}}} \sum_{n=1}^{\tau_{\mathrm{est}} f_{\mathrm{s}}}\left|y_{\mathrm{ST}}[n]\right|^{2} \text {. }
$$

\footnotetext{
${ }^{4}$ Therefore, the sensing phase incorporates the estimation phase, see Fig. 2.
} 


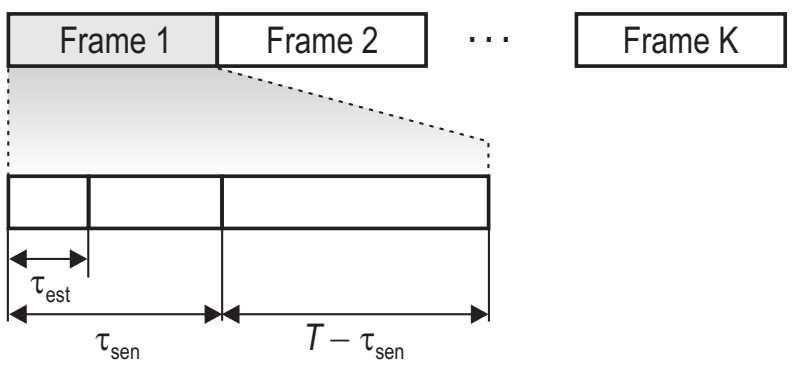

Fig. 2. An illustration of the proposed frame structure for an interweave system depicting the estimation phase and the sensing phase for the sensing channel.

$\hat{P}_{\mathrm{Rx}, \mathrm{ST}}$ determined in (10) using $\tau_{\text {est }} f_{\mathrm{s}}$ samples follows a central chi-squared distribution $\mathcal{X}^{2}$ [27]. The cumulative distribution function (CDF) of $\hat{P}_{\mathrm{Rx}, \mathrm{ST}}$ is given by

$$
F_{\hat{P}_{\mathrm{R}, \mathrm{ST}}}(x)=1-\Gamma\left(\frac{\tau_{\text {est }} f_{\mathrm{s}}}{2}, \frac{\tau_{\mathrm{est}} f_{\mathrm{s}} x}{2 P_{\mathrm{Rx}, \mathrm{ST}}}\right) .
$$

2) Estimation of access channel $\left(h_{s}\right)$ : The signal received from the ST undergoes matched filtering and demodulation at the SR, hence, it is reasonable to employ pilot based estimation for $h_{\mathrm{s}}$. Unlike received power based estimation, pilot based estimation renders a direct estimation of the channel. Now, to accomplish pilot based estimation, the SR aligns itself to pilot symbols transmitted by the ST. Under $\mathcal{H}_{0}$, the discrete and real pilot symbols at the output of the demodulator is given by [20]

$$
p[n]=\sqrt{E_{\mathrm{s}}} h_{\mathrm{s}}+w[n],
$$

where $E_{\mathrm{s}}$ denotes the pilot energy. Without loss of generality, the pilot symbols are considered to be +1 . The maximum likelihood estimate, representing a sample average of $N_{\mathrm{s}}$ pilot symbols, is given by [19]

$$
h_{\mathrm{s}}=\hat{h}_{\mathrm{s}}+\underbrace{\frac{\sum_{n}^{N_{\mathrm{s}}} p[n]}{2 N_{\mathrm{s}}}},
$$

where $\epsilon$ denotes the estimation error. The estimate $\hat{h}_{\mathrm{s}}$ is unbiased, efficient and achieves a Cramér-Rao bound with equality, with variance $\mathbb{E}\left[\left|h_{\mathrm{s}}-\hat{h}_{\mathrm{s}}\right|^{2}\right]=\sigma_{w}^{2} /\left(2 N_{\mathrm{s}}\right)$ [20]. Consequently, $\hat{h}_{\mathrm{s}}$ conditioned on $h_{\mathrm{s}}$ follows a Gaussian distribution.

$$
\hat{h}_{\mathrm{s}} \mid h_{\mathrm{s}} \sim \mathcal{N}\left(h_{\mathrm{s}}, \frac{\sigma_{w}^{2}}{2 N_{\mathrm{s}}}\right) .
$$

As a result, the power gain $\left|\hat{h}_{\mathrm{s}}\right|^{2}$ follows a non-central chisquared $\left(\mathcal{X}_{1}^{2}\right)$ distribution with 1 degree of freedom and noncentrality parameter $\lambda=\frac{2 N_{\mathrm{s}}\left|h_{\mathrm{s}}\right|^{2}}{\sigma_{w}^{2}}$.

3) Estimation of interference channel $\left(h_{p, 2}\right)$ : Analog to sensing channel, the SR performs received power based estimation by listening to the transmission from the PT. The knowledge of $h_{\mathrm{p}, 2}$ is required to characterize interference from the PT. Under $\mathcal{H}_{1}$, the discrete signal model at the $\mathrm{SR}$ is given as

$$
y_{\mathrm{SR}}[n]=h_{\mathrm{p}, 2} \cdot x_{\mathrm{PT}}[n]+w[n] \text {. }
$$

The received power at the SR from the PT given by

$$
\hat{P}_{\mathrm{Rx}, \mathrm{SR}}=\frac{1}{N_{\mathrm{p}, 2}} \sum_{n=1}^{N_{\mathrm{p}, 2}}\left|y_{\mathrm{SR}}[n]\right|^{2},
$$

follows a $\mathcal{X}^{2}$ distribution, where $N_{\mathrm{p}, 2}$ corresponds to the number of samples used for estimation.

\section{Validation}

It is now clear that the estimates $\hat{P}_{\mathrm{Rx}, \mathrm{ST}},\left|\hat{h}_{\mathrm{s}}\right|^{2}$ and $\hat{P}_{\mathrm{Rx}, \mathrm{SR}}$ exhibit the knowledge corresponding to the involved channels, however, it is essential to validate them, mainly $\hat{P}_{\mathrm{Rx}, \mathrm{ST}}$ and $\hat{P}_{\mathrm{Rx}, \mathrm{SR}}$. In this context, it is necessary to ensure the presence of the PU signal $\left(\mathcal{H}_{1}\right)$ for that particular frame. In this direction, Chavali et al. [21] recently proposed a detection followed by the estimation of the signal to noise ratio, while [35] implemented a blind technique for estimating signal power of non-coherent PU signals. In this paper, we propose a different methodology, according to which, we apply a coarse detection $^{5}$ on the estimates $\hat{P}_{\mathrm{Rx}, \mathrm{ST}}, \hat{P}_{\mathrm{Rx}, \mathrm{SR}}$ at the end of the estimation phase $\tau_{\text {est }}$. Through an appropriate selection of the time interval $\tau_{\text {est }}$ (for instance, $\tau_{\text {est }} \in[1,10] \mathrm{ms}$ ) during the system design, the reliability of the coarse detection can be ensured. With the existence of a separate control channel such as cognitive pilot channel, the reliability of the coarse detection can be further enhanced by exchanging the detection results between the ST and the SR.

Since the estimation and the coarse detection processes in our proposed method are equivalent in terms of their mathematical operations (which include magnitude squared and summation), we consider the validity of the channel estimates with certain reliability and without comprising the complexity of the estimators employed by the secondary system. Moreover, by performing a joint estimation and (coarse) detection, we propose an efficient way of utilizing the time resources within the frame duration. The ST considers these estimates to determine a suitable sensing time based on the sensing-throughput tradeoff such that the desired detector's performance is ensured. At the end of the detection phase, we carry out fine detection ${ }^{6}$ of the PU signals, thereby improving the performance of the detector.

\section{Assumptions and Approximations}

To simplify the analysis and sustain analytical tractability for the proposed approach, several assumptions considered in the paper are summarized as follows:

- We consider that all transmitted signals are subjected to distance dependent path loss and small scale fading gain. With no loss of generality, we consider that the channel gains include distance dependent path loss and small scale gain. Moreover, the coherence time for the channel gain is considered to be greater than the frame duration ${ }^{7}$.

- We assume the perfect knowledge of the noise power in the system, however, the uncertainty in noise power can be captured as a bounded interval [28]. Inserting this

\footnotetext{
${ }^{5}$ For the coarse detection, an energy detection is employed whose threshold can be determined by means of a constant false alarm rate.

${ }^{6}$ In accordance with the proposed frame structure in Fig. 2, fine detection represents the main detection which also includes the samples acquired during the estimation phase.

${ }^{7}$ In the scenarios where the coherence time exceeds the frame duration, in such cases, our characterization depicts a lower performance bound.
} 
interval in the derived expressions, refer to Section IV, the performance of the IS can be expressed in terms of the upper and the lower bounds.

- For all degrees of freedom, $\mathcal{X}_{1}^{2}$ distribution can be approximated by Gamma distribution [36]. The parameters of the Gamma distribution are obtained by matching the first two central moments to those of $\mathcal{X}_{1}^{2}$.

\section{THEORETICAL ANALYSIS}

At this stage, it is evident that the variation due to imperfect channel knowledge translates to the variations of the performance parameters $\mathrm{P}_{\mathrm{d}}, \mathrm{C}_{0}$ and $\mathrm{C}_{1}$, which are fundamental to sensing-throughput tradeoff. Below, we capture these variations by characterizing their cumulative distribution functions $F_{\mathrm{P}_{\mathrm{d}}}, F_{\mathrm{C}_{0}}$ and $F_{\mathrm{C}_{1}}$, respectively.

Lemma 1: The cumulative distribution function of $\mathrm{P}_{\mathrm{d}}$ is characterized as

$$
F_{\mathrm{P}_{\mathrm{d}}}(x)=1-\Gamma\left(\frac{\tau_{\mathrm{est}} f_{\mathrm{s}}}{2}, \frac{\tau_{\mathrm{est}} f_{\mathrm{s}} \tau_{\mathrm{sen}} f_{\mathrm{s}} \mu}{4 P_{\mathrm{Rx}, \mathrm{ST}} \Gamma^{-1}\left(x, \frac{\tau_{\mathrm{sen}} f_{\mathrm{s}}}{2}\right)}\right),
$$

where $\Gamma^{-1}(\cdot, \cdot)$ is inverse function of regularized incomplete upper Gamma function [29].

Proof: The cumulative distribution function of $\mathrm{P}_{\mathrm{d}}$ is defined as

$$
F_{\mathrm{P}_{\mathrm{d}}}(x)=\mathbb{P}\left(\mathrm{P}_{\mathrm{d}}\left(\mu, \tau_{\mathrm{sen}}, \hat{P}_{\mathrm{Rx}, \mathrm{ST}}\right) \leq x\right) .
$$

Using (4)

$$
\begin{gathered}
=\mathbb{P}\left(\Gamma\left(\frac{\tau_{\mathrm{sen}} f_{\mathrm{s}}}{2}, \frac{\tau_{\mathrm{sen}} f_{\mathrm{s}} \mu}{2 \hat{P}_{\mathrm{Rx}, \mathrm{ST}}}\right) \leq x\right), \\
=1-\mathbb{P}\left(\hat{P}_{\mathrm{Rx}, \mathrm{ST}} \geq \frac{\mu \tau_{\mathrm{sen}} f_{\mathrm{s}}}{2 \Gamma^{-1}\left(x, \frac{\tau_{\mathrm{sen}} f_{\mathrm{s}}}{2}\right)}\right) .
\end{gathered}
$$

Replacing the cumulative distribution function of $\hat{P}_{\mathrm{RX}, \mathrm{ST}}$ in (20), we obtain an expression of $F_{\mathrm{P}_{\mathrm{d}}}$.

Lemma 2: The cumulative distribution function of $\mathrm{C}_{0}$ is defined as

$$
F_{\mathrm{C}_{0}}(x)=\int_{0}^{x} f_{\mathrm{C}_{0}}(t) d t
$$

where

$$
f_{\mathrm{C}_{0}}(x)=2^{x} \ln 2 \frac{\left(2^{x}-1\right)^{a_{1}-1}}{\Gamma\left(a_{1}\right) b_{1}^{a_{1}}} \exp \left(-\frac{2^{x}-1}{b_{1}}\right),
$$

and

$$
\begin{aligned}
& a_{1}=\frac{\left(\frac{\sigma_{w}^{4}}{2 N_{\mathrm{s}} P_{\mathrm{T}, \mathrm{ST}}}+\left|h_{\mathrm{s}}\right|^{2}\right)^{2}}{\frac{\sigma_{w}^{4}}{2 N_{\mathrm{s}} P_{\mathrm{T}, \mathrm{ST}}}\left(2 \frac{\sigma_{w}^{4}}{2 N_{\mathrm{s}} P_{\mathrm{T} \times, S \mathrm{~T}}}+4\left|h_{\mathrm{s}}\right|^{2}\right)} \text { and } \\
& b_{1}=\frac{\frac{\sigma_{w}^{4}}{2 N_{\mathrm{s}} P_{\mathrm{T}, \mathrm{ST}}}\left(2 \frac{\sigma_{w}^{4}}{2 N_{\mathrm{s}} P_{\mathrm{TX}, \mathrm{ST}}}+4\left|h_{\mathrm{s}}\right|^{2}\right)}{\left(\frac{\sigma_{w}^{4}}{2 N_{\mathrm{s}} P_{\mathrm{T}, S \mathrm{ST}}}+\left|h_{\mathrm{s}}\right|^{2}\right)} .
\end{aligned}
$$

(a)

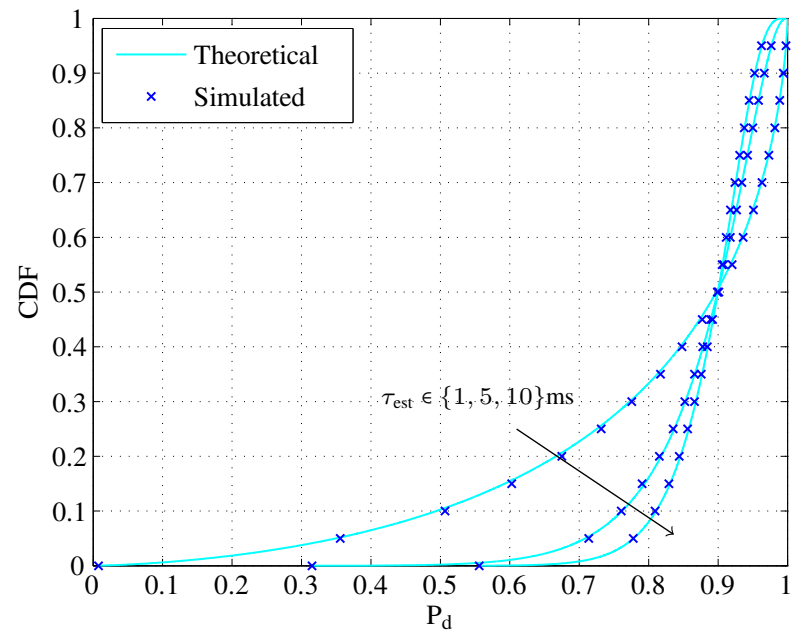

(b)

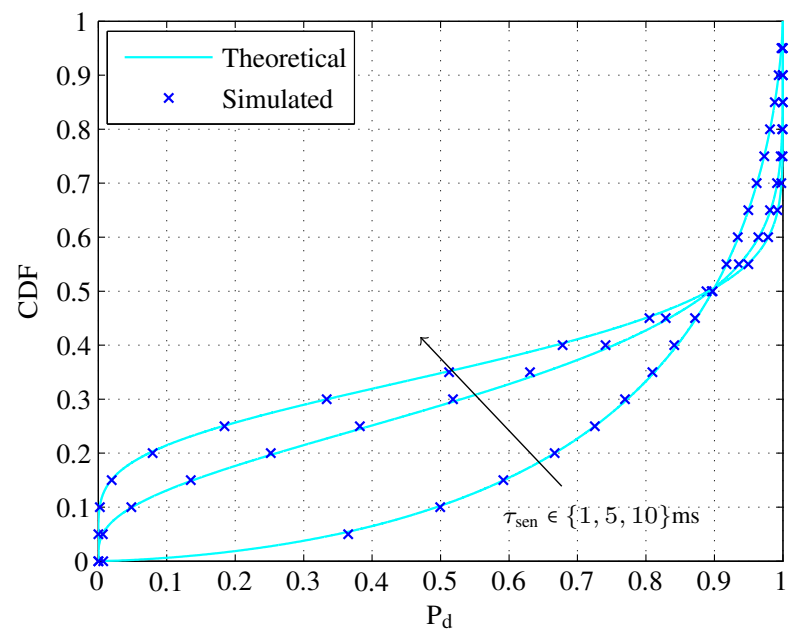

Fig. 3. $\mathrm{CDF}$ of $\mathrm{P}_{\mathrm{d}}$ for different $\tau_{\text {est }}$ and $\tau_{\text {sen. }}$. (a) $\tau_{\text {est }} \in\{1,5,10\} \mathrm{ms}$ and $\tau_{\text {sen }}=1 \mathrm{~ms}$, (b) $\tau_{\text {est }}=1 \mathrm{~ms}$ and $\tau_{\text {sen }} \in\{1,5,10\} \mathrm{ms}$.

Proof: Following the probability density function (pdf) of $\left|\hat{h}_{\mathrm{S}}\right|^{2}$ in (14), the pdf $\left|\hat{h}_{\mathrm{S}}\right|^{2} \frac{P_{\mathrm{T} \times S \mathrm{ST}}}{\sigma_{w}^{2}}$ is given by

$$
\begin{array}{r}
f_{\frac{\left|\hat{h}_{\mathrm{s}}\right|^{2} P_{\mathrm{T} \times \mathrm{ST}}}{\sigma_{w}^{2}}}(x)=\frac{2 N_{\mathrm{s}} P_{\mathrm{Tx}, \mathrm{ST}}}{\sigma_{w}^{4}} \frac{1}{2} \exp \left[-\frac{1}{2}\left(x \frac{\sigma_{w}^{4}}{2 N_{\mathrm{s}} P_{\mathrm{Tx}, \mathrm{ST}}}+\lambda\right)\right] \times \\
\left(\frac{x}{\lambda} \frac{\sigma_{w}^{4}}{2 N_{\mathrm{s}} P_{\mathrm{T} \mathrm{S}, \mathrm{ST}}}\right)^{\frac{N_{\mathrm{s}}}{4}-\frac{1}{2}} I_{\frac{N_{\mathrm{s}}}{2}-1}\left(\sqrt{\lambda x \frac{\sigma_{w}^{4}}{2 N_{\mathrm{S}} P_{\mathrm{Tx}, \mathrm{ST}}}}\right),
\end{array}
$$

where $I_{(\cdot)}(\cdot)$ represents the modified Bessel function of first kind [29]. Approximating $\mathcal{X}_{1}^{2}(\cdot, \cdot)$ with Gamma distribution $\Gamma\left(a_{1}, b_{1}\right)[36]$ gives

$$
f_{\frac{\left|\hat{h}_{\mathrm{s}}\right|^{2} P_{\mathrm{T} X S \mathrm{ST}}}{\sigma_{w}^{2}}} \approx \frac{1}{\Gamma\left(a_{1}\right)} \frac{x^{a_{1}-1}}{b_{1}^{a_{1}}} \exp \left(-\frac{x}{b_{1}}\right),
$$

where the parameters $a_{1}$ and $b_{1}$ in (24) are determined by comparing the first two central moments of the two distributions. Finally, by substituting the expression of $\mathrm{C}_{0}$ in (8) yields (22).

Lemma 3: The cumulative distribution function of $\mathrm{C}_{1}$ is 


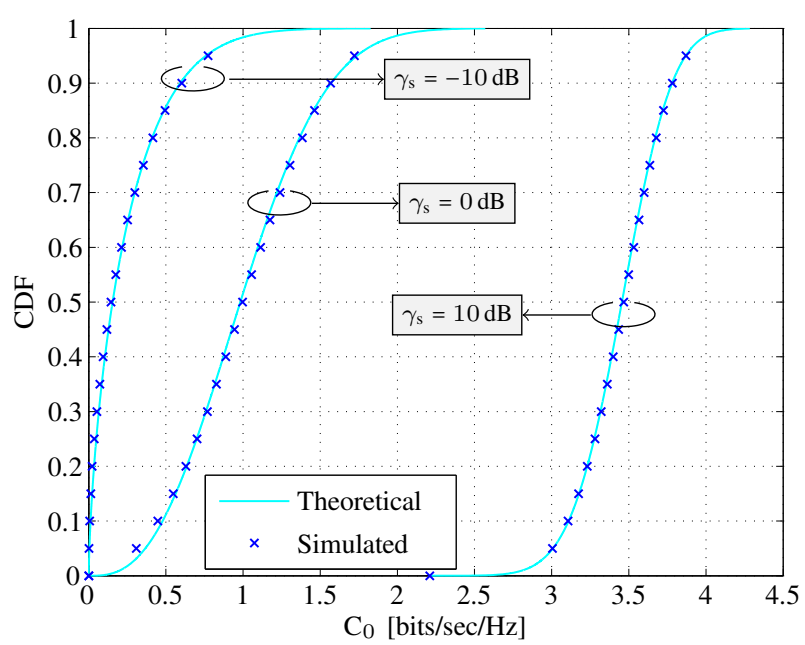

Fig. 4. $\mathrm{CDF}$ of $\mathrm{C}_{0}$ for different values of $\gamma_{\mathrm{s}} \in\{-10,0,10\} \mathrm{dB}$.

given by

$$
F_{\mathrm{C}_{1}}(x)=\int_{0}^{x} f_{\mathrm{C}_{1}}(t) d t
$$

where

$f_{\mathrm{C}_{1}}(x)=2^{x} \ln 2 \frac{\left(2^{x}-1\right)^{a_{1}-1} \Gamma\left(a_{1}+a_{2}\right)}{\Gamma\left(a_{1}\right) \Gamma\left(a_{2}\right) b_{1}^{a_{1}} b_{2}^{a_{2}}}\left(\frac{1}{b_{2}}+\frac{2^{x}-1}{b_{1}}\right)^{\left(a_{1}+a_{2}\right)}$,

and

$$
a_{2}=\frac{N_{\mathrm{p}, 2}}{2} \text { and } b_{2}=\frac{2 P_{\mathrm{Rx}, \mathrm{SR}}}{\sigma_{w}^{2} N_{\mathrm{p}, 2}}
$$

where $a_{1}$ and $b_{1}$ are defined in (23).

Proof: See Appendix A.

The theoretical expressions of the distribution functions depicted in Lemma 1, Lemma 2 and Lemma 3 are validated by means of simulations in Fig. 3, Fig. 4 and Fig. 5, respectively, with different choices of system parameters, these include $\tau_{\text {est }} \in\{1,5,10\} \mathrm{ms}, \tau_{\text {sen }}=\{1,5,10\} \mathrm{ms}, \gamma_{\mathrm{s}} \in\{-10,0,10\} \mathrm{dB}$ and $\gamma_{\mathrm{p}, 2} \in\{-10,0,10\} \mathrm{dB}$.

\section{A. Sensing-throughput tradeoff}

Here, we establish sensing-throughput tradeoff for the estimation model that includes the estimation time and incorporates variations in the performance parameter. Most importantly, to restrain the harmful interference at the PR due to the variations in the detection probability, we propose two new PU constraints at the PR, namely, an average constraint and an outage constraint on the detection probability. Based on these constraints, we characterize the sensing-throughput tradeoff for the IS.

Theorem 1: Subject to an average constraint on $\mathrm{P}_{\mathrm{d}}$ at the (a)

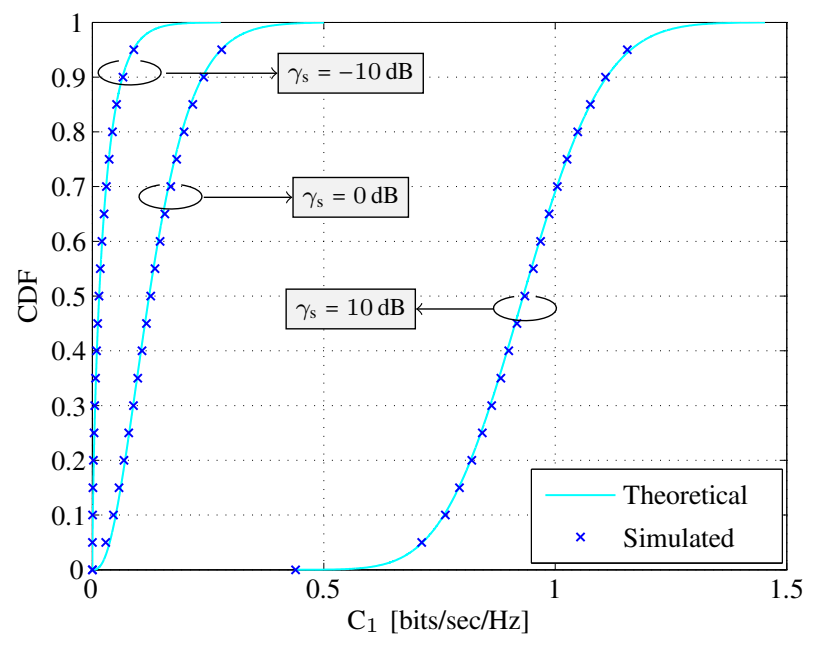

(b)

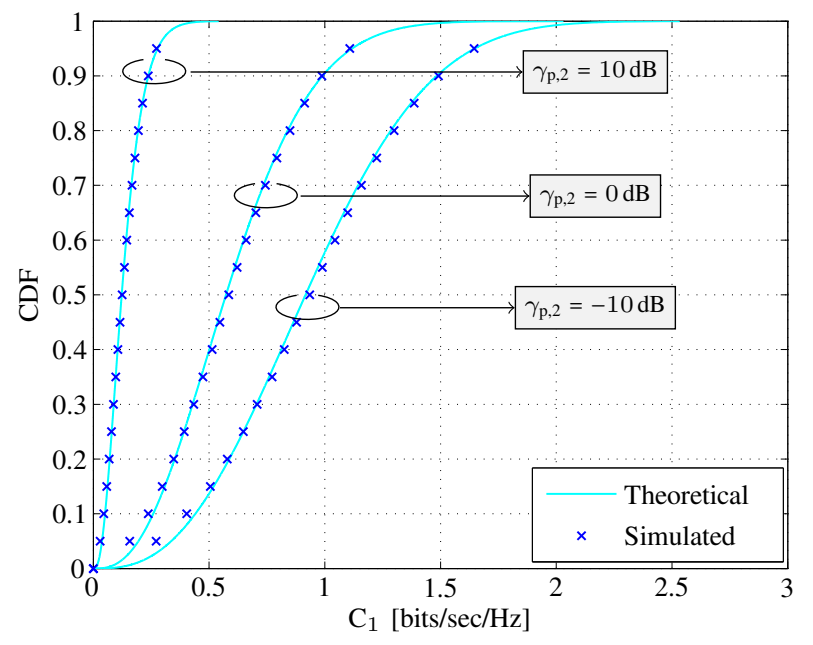

Fig. 5. $\mathrm{CDF}$ of $\mathrm{C}_{1}$ for different $\gamma_{\mathrm{s}}$ and $\gamma_{\mathrm{p}, 2}$. (a) $\gamma_{\mathrm{s}} \in\{-10,0,10\} \mathrm{dB}$ and $\gamma_{\mathrm{p}, 2}=10 \mathrm{~dB}$, (b) $\gamma_{\mathrm{s}}=0 \mathrm{~dB}$ and $\gamma_{\mathrm{p}, 2} \in\{-10,0,10\} \mathrm{dB}$.

PR, the sensing-throughput tradeoff is given by

$$
\begin{aligned}
R_{\mathrm{s}}\left(\tilde{\tau}_{\mathrm{est}}, \tilde{\tau}_{\text {sen }}\right) & =\max _{\tau_{\text {est }}, \tau_{\text {sen }}} \mathbb{E}_{\mathrm{P}_{\mathrm{d}}, \mathrm{C}_{0}, \mathrm{C}_{1}}\left[R_{\mathrm{s}}\left(\tau_{\mathrm{est}}, \tau_{\text {sen }}\right)\right] \\
& =\frac{T-\tau_{\text {sen }}}{T}\left[\mathbb{E}_{\mathrm{C}_{0}}\left[\mathrm{C}_{0}\right]\left(1-\mathrm{P}_{\mathrm{fa}}\right) \mathbb{P}\left(\mathcal{H}_{0}\right)+\right. \\
& \left.\mathbb{E}_{\mathrm{C}_{1}}\left[\mathrm{C}_{1}\right]\left(1-\mathbb{E}_{\mathrm{P}_{\mathrm{d}}}\left[\mathrm{P}_{\mathrm{d}}\right]\right) \mathbb{P}\left(\mathcal{H}_{1}\right)\right] \\
\text { s.t. } & \mathbb{E}_{\mathrm{P}_{\mathrm{d}}}\left[\mathrm{P}_{\mathrm{d}}\right] \leq \overline{\mathrm{P}}_{\mathrm{d}}, \\
\text { s.t. } & 0<\tau_{\text {est }} \leq \tau_{\text {sen }} \leq T
\end{aligned}
$$

where $\mathbb{E}_{P_{d}}[\cdot]$ represents the expectation with respect to $P_{d}$, $\mathbb{E}_{\mathrm{P}_{\mathrm{d}}, \mathrm{C}_{0}, \mathrm{C}_{1}}[\cdot]$ denotes the expectation with respect to $\mathrm{P}_{\mathrm{d}}, \mathrm{C}_{0}$ and $C_{1}$. Unlike (7), $\bar{P}_{d}$ in (28) represents the constraint on expected detection probability.

Proof: See Appendix B. For simplification, the proof of Theorem 1 is included in the proof of Theorem 2.

Theorem 2: Subject to an outage constraint on $\mathrm{P}_{\mathrm{d}}$ at the PR, 
the sensing-throughput tradeoff is given by

$$
\begin{aligned}
R_{\mathrm{s}}\left(\tilde{\tau}_{\text {est }}, \tilde{\tau}_{\text {sen }}\right) & =\max _{\tau_{\text {est }}, \tau_{\text {sen }}} \mathbb{E}_{\mathrm{P}_{\mathrm{d}}, \mathrm{C}_{0}, \mathrm{C}_{1}}\left[R_{\mathrm{s}}\left(\tau_{\mathrm{est}}, \tau_{\text {sen }}\right)\right], \\
& =\frac{T-\tau_{\text {sen }}}{T}\left[\mathbb{E}_{\mathrm{C}_{0}}\left[\mathrm{C}_{0}\right]\left(1-\mathrm{P}_{\mathrm{fa}}\right) \mathbb{P}\left(\mathcal{H}_{0}\right)+\right. \\
& \left.\mathbb{E}_{\mathrm{C}_{1}}\left[\mathrm{C}_{1}\right]\left(1-\mathbb{E}_{\mathrm{P}_{\mathrm{d}}}\left[\mathrm{P}_{\mathrm{d}}\right]\right) \mathbb{P}\left(\mathcal{H}_{1}\right)\right] \\
\text { s.t. } & \mathbb{P}\left(\mathrm{P}_{\mathrm{d}} \leq \overline{\mathrm{P}}_{\mathrm{d}}\right) \leq \kappa \\
\text { s.t. } & 0<\tau_{\text {est }} \leq \tau_{\text {sen }} \leq T,
\end{aligned}
$$

where $\kappa$ represents the outage constraint.

Proof: See Appendix B.

In contrast to the ideal model, the sensing-throughput tradeoff investigated by the estimation model (refer to Theorems 1 and 2) incorporates the imperfect channel knowledge, in this context, the performance characterization considered by the proposed framework are closer to the realistic situations.

Remark 1: Herein, based on the estimation model, we establish a fundamental relation between estimation time (regulates the variation in the detection probability according to the PU constraint), sensing time (represents the detector performance) and achievable throughput, this relationship is characterized as estimation-sensing-throughput tradeoff. Based on this tradeoff, we determine the suitable estimation $\tau_{\text {est }}=\tilde{\tau}_{\text {est }}$ and sensing time $\tau_{\text {sen }}=\tilde{\tau}_{\text {sen }}$ that attains a maximum achievable throughput $R_{\mathrm{s}}\left(\tilde{\tau}_{\text {est }}, \tilde{\tau}_{\text {sen }}\right)$ for the IS.

Corollary 1: Theorems 1 and 2 consider the optimization of the average throughput to incorporate the effect of variations due to channels estimation, and subsequently determine the suitable sensing and the suitable estimation time. Here, we investigate an alternative approach to the optimization problem described in (6) to capture these variations, whereby for a certain estimation time $\tau_{\text {est }}$, the suitable sensing time subject to the average constraint is determined as

$$
\begin{aligned}
\tilde{\tau}_{\text {sen }} & =\underset{\tau_{\text {sen }}}{\operatorname{argmax}} R_{\mathrm{s}}\left(\tau_{\text {est }}, \tau_{\text {sen }}\right), \\
& =\frac{T-\tau_{\text {sen }}}{T}\left[\mathrm{C}_{0}\left(1-\mathrm{P}_{\mathrm{fa}}\right) \mathbb{P}\left(\mathcal{H}_{0}\right)+\mathrm{C}_{1}\left(1-\mathrm{P}_{\mathrm{d}}\right) \mathbb{P}\left(\mathcal{H}_{1}\right)\right], \\
\text { s.t. } & \mathbb{E}_{\mathrm{P}_{\mathrm{d}}}\left[\mathrm{P}_{\mathrm{d}}\right] \leq \overline{\mathrm{P}}_{\mathrm{d}}, \\
\text { s.t. } & 0<\tau_{\text {est }} \leq \tau_{\text {sen }} \leq T .
\end{aligned}
$$

Similarly, the suitable sensing time subject to the outage constraint is determined as

$$
\begin{aligned}
\tilde{\tau}_{\text {sen }} & =\underset{\tau_{\text {sen }}}{\operatorname{argmax}} R_{\mathrm{s}}\left(\tau_{\mathrm{est}}, \tau_{\text {sen }}\right) \\
& =\frac{T-\tau_{\text {sen }}}{T}\left[\mathrm{C}_{0}\left(1-\mathrm{P}_{\mathrm{fa}}\right) \mathbb{P}\left(\mathcal{H}_{0}\right)+\mathrm{C}_{1}\left(1-\mathrm{P}_{\mathrm{d}}\right) \mathbb{P}\left(\mathcal{H}_{1}\right)\right] \\
\text { s.t. } & \mathbb{P}\left(\mathrm{P}_{\mathrm{d}} \leq \overline{\mathrm{P}}_{\mathrm{d}}\right) \leq \kappa \\
\text { s.t. } & 0<\tau_{\text {est }} \leq \tau_{\text {sen }} \leq T .
\end{aligned}
$$

In contrast to (28) and (30), the suitable sensing time evaluated in (32) and (33) entails the variations due to channel estimation. Hence, the secondary throughput subject to the average and the outage constraints captures the variations in the suitable sensing time and the performance parameters is
TABLE II

PARAMETERS FOR NUMERICAL ANALYSIS

\begin{tabular}{c||c}
\hline \multicolumn{1}{c||}{ Parameter } & Value \\
\hline \hline$f_{\mathrm{s}}$ & $1 \mathrm{MHz}$ \\
\hline$\left|h_{\mathrm{p}, 1}\right|^{2},\left|h_{\mathrm{p}, 2}\right|^{2}$ & $-100 \mathrm{~dB}$ \\
\hline$\left|h_{\mathrm{s}}\right|^{2}$ & $-80 \mathrm{~dB}$ \\
\hline$T$ & $100 \mathrm{~ms}$ \\
\hline$\overline{\mathrm{P}}_{\mathrm{d}}$ & 0.9 \\
\hline$\kappa$ & 0.05 \\
\hline$\sigma_{w}^{2}$ & $-100 \mathrm{dBm}$ \\
\hline$\gamma_{\mathrm{p}, 1}$ & $-10 \mathrm{~dB}$ \\
\hline$\gamma_{\mathrm{p}, 2}$ & $-10 \mathrm{~dB}$ \\
\hline$\gamma_{\mathrm{s}}$ & $10 \mathrm{~dB}$ \\
\hline$\sigma_{x}^{2}=P_{\mathrm{Tx}, \mathrm{PT}}$ & $-10 \mathrm{dBm}$ \\
\hline$P_{\mathrm{T}, \mathrm{ST}}$ & $-10 \mathrm{dBm}$ \\
\hline $\mathbb{P}\left(\mathcal{H}_{1}\right)=1-\mathbb{P}\left(\mathcal{H}_{0}\right)$ & 0.2 \\
\hline$\tau_{\mathrm{est}}$ & $5 \mathrm{~ms}$ \\
\hline$N_{\mathrm{s}}$ & 10 \\
\hline$N_{\mathrm{p}, 2}$ & 1000 \\
\hline &
\end{tabular}

determined as

$$
\mathbb{E}_{\mathrm{P}_{\mathrm{d}}, \mathrm{C}_{0}, \mathrm{C}_{1}, \tilde{\tau}_{\text {sen }}}\left[R_{\mathrm{s}}\left(\tau_{\text {est }}, \tilde{\tau}_{\text {sen }}\right)\right],
$$

where $\mathbb{E}_{\mathrm{P}_{\mathrm{d}}, \mathrm{C}_{0}, \mathrm{C}_{1}, \tilde{\tau}_{\mathrm{sen}}}[\cdot]$ corresponds to an expection over $\mathrm{P}_{\mathrm{d}}, \mathrm{C}_{0}, \mathrm{C}_{1}, \tilde{\tau}_{\text {sen }}$. Following Remark 1, we further optimize the average throughput, defined in (34), over the estimation time

$$
R_{\mathrm{S}}\left(\tilde{\tau}_{\mathrm{est}}, \tilde{\tau}_{\mathrm{sen}}\right)=\max _{\tau_{\mathrm{est}}} \mathbb{E}_{\mathrm{P}_{\mathrm{d}}, \mathrm{C}_{0}, \mathrm{C}_{1}, \tilde{\tau}_{\text {sen }}}\left[R_{\mathrm{S}}\left(\tau_{\mathrm{est}}, \tilde{\tau}_{\mathrm{sen}}\right)\right] .
$$

In this way, we establish an estimation-sensing-throughput tradeoff for the alternative approach to determine the suitable estimation time.

Remark 2: Complementing the analysis in [13], it is complicated to obtain a closed-form expression of $\tilde{\tau}_{\text {sen }}$, thereby rendering the analytical tractability of its distribution function difficult. In view of this, we capture the performance of the alternative approach by means of simulations.

\section{Numerical Results}

Here, we investigate the performance of the IS based on the proposed approach. To accomplish this: (i) we perform simulations to validate the expressions obtained, (ii) we analyze the performance loss incurred due to the estimation. In this regard, we consider the ideal model to benchmark and evaluate the performance loss, (iii) we establish mathematical justification to the considered approximations. Although the expressions derived in this paper depicting the sensing-throughput analysis are general and applicable to all CR systems, the parameters are selected in such a way that they closely relate to the deployment scenario described in Fig. 1. Unless stated explicitly, the choice of the parameters given in Table II is considered for the analysis.

At first, we analyze the performance of the IS in terms of sensing-throughput tradeoff corresponding to the ideal model (IM) and estimation model (EM) by fixing $\tau_{\text {est }}=5 \mathrm{~ms}$, refer to Fig. 6. In contrast to constraint on $\mathrm{P}_{\mathrm{d}}$ for the ideal model, we employ average constraint (EM-AC) and outage 


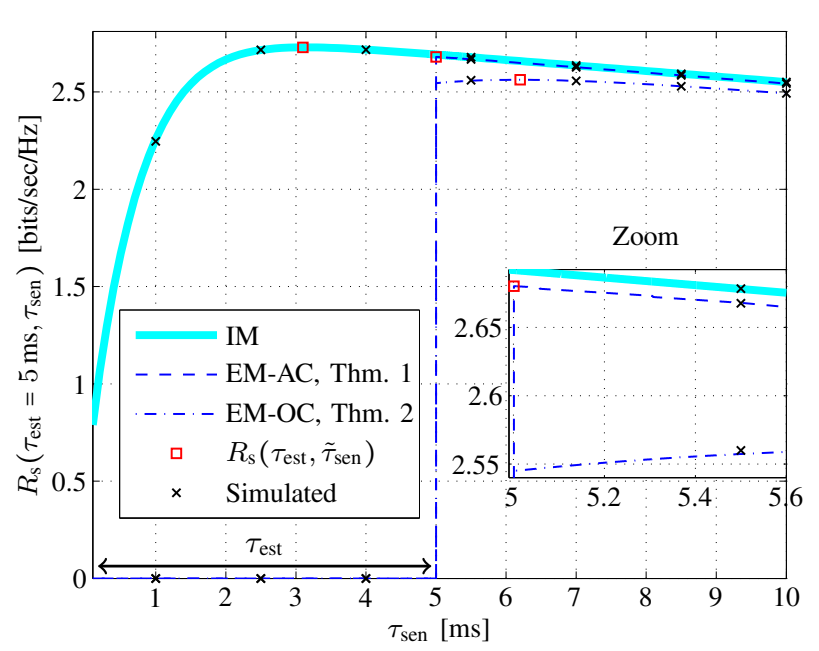

Fig. 6. Sensing-throughput tradeoff for the ideal model (IM) and estimation model $(\mathrm{EM}), \gamma_{\mathrm{p}, 1}=-10 \mathrm{~dB}, \tau_{\mathrm{est}}=5 \mathrm{~ms}$ and $\kappa=0.05$.

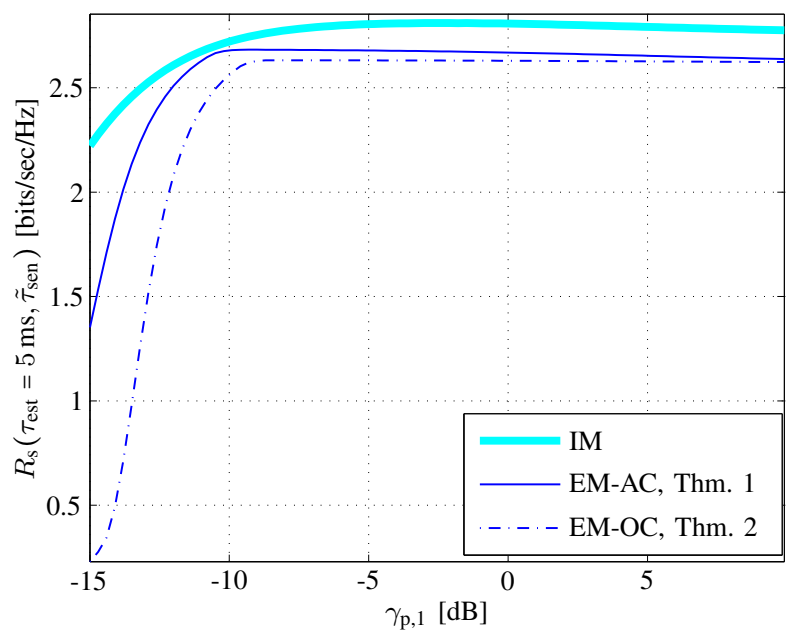

Fig. 7. Achievable throughput versus the $\gamma_{\mathrm{p}, 1}$ with $\tau_{\mathrm{est}}=5 \mathrm{~ms}$.

constraint (EM-OC) for the proposed estimation model. With the inclusion of received power based estimation in the frame structure, the ST achieves no throughput at the SR for the interval $\tau_{\text {est. }}$. For the given cases, namely, IM, EM-AC and EM-OC, a suitable sensing time that results in a maximum throughput $R_{\mathrm{s}}\left(\tau_{\mathrm{est}}=5 \mathrm{~ms}, \tilde{\tau}_{\mathrm{sen}}\right)$ is determined. Apart form that, a performance degradation is depicted in terms of the achievable throughput, refer to Fig. 6 . For $\kappa=0.05$, it is observed that the outage constraint is more sensitive to the performance loss in comparison to average constraint. It is clear that the analysis illustrated in Fig. 6 is obtained for a certain choice of system parameters, particularly $\gamma_{\mathrm{p}, 1}=-10 \mathrm{~dB}, \tau_{\mathrm{est}}=5 \mathrm{~ms}$ and $\kappa=0.05$. To acquire more insights, we consider the effect of variation of these parameters on the performance of IS, subsequently.

Hereafter, for the analysis, we consider the theoretical expressions and choose to operate at a suitable sensing time. Next, we capture the variation in the achievable throughput against the received signal to noise ratio $\gamma_{\mathrm{p}, 1}$ at the ST with $\tau_{\text {est }}=5 \mathrm{~ms}$, refer to Fig. 7. For $\gamma_{\mathrm{p}, 1}<-10 \mathrm{~dB}$, the estimation model incurs a significant performance loss. This clearly reveals that the ideal model overestimates the performance (a)

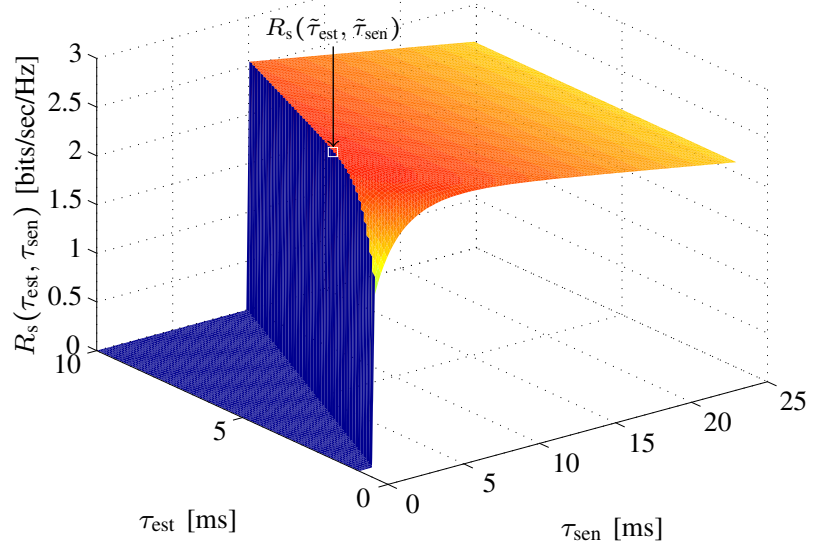

(b)

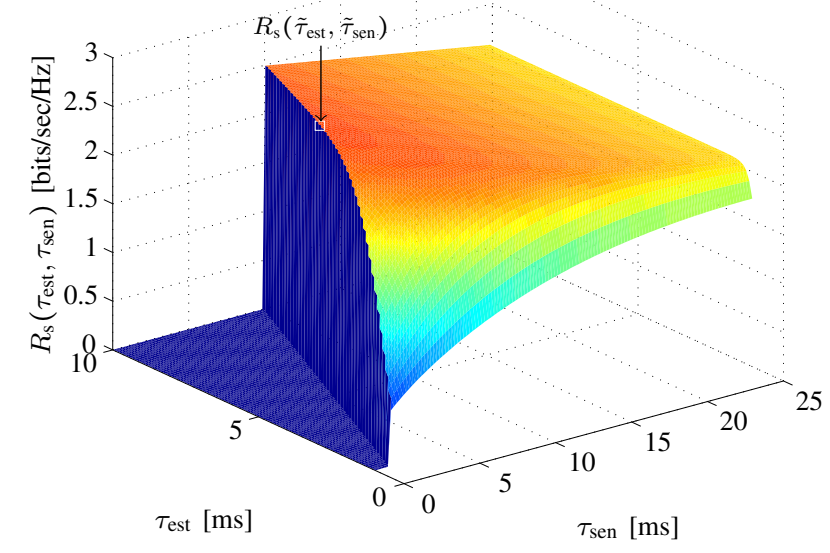

Fig. 8. Estimation-sensing-throughput tradeoff for the estimation model for (a) average constraint and (b) outage constraint with $\kappa=0.05$.

of IS. From the previous discussion, it is concluded that the inclusion of average and outage constraints (depicted by the proposed framework) preclude the excessive interference at the PR arising due to channel estimation without considerably degrading the performance of the IS.

Upon maximizing the secondary throughput, it is interesting to analyze the variation of the achievable throughput with the estimation time. Corresponding to the estimation model, Fig. 8 illustrates a tradeoff among the estimation time, the sensing time and the throughput, refer to Remark 1. From Fig. 8, it can be noticed that the function $R_{\mathrm{s}}\left(\tau_{\text {est }}, \tau_{\text {sen }}\right)$ is well-behaved in the region $0<\tau_{\text {est }} \leq \tau_{\text {sen }} \leq T$ and consists of a global maximum. This tradeoff depicted by the proposed framework, presented in Fig. 9, can be explained from the fact that low values of estimation time result in large variations in $P_{d}$. To counteract and satisfy the average and the outage constraints, the corresponding thresholds shift to a lower value. This causes an increase in $\mathrm{P}_{\mathrm{fa}}$, thereby increasing the sensing-throughput curvature. As a result, the suitable sensing time is obtained at a higher value. However, beyond a certain value $\left(\tilde{\tau}_{\text {est }}\right)$, a further increase in estimation time slightly contributes to performance improvement and largely consumes the time resources. As a 


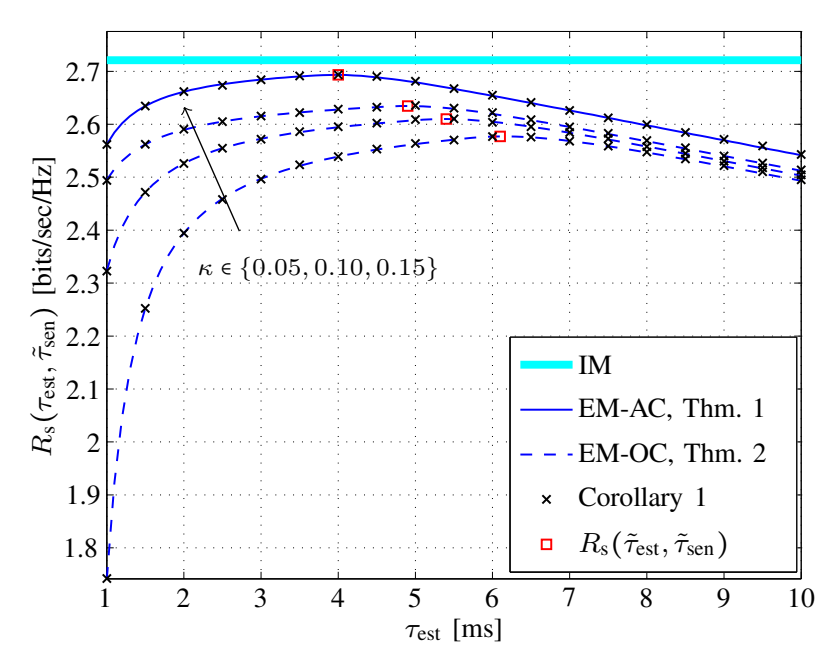

Fig. 9. Estimation-sensing-throughput tradeoff for the average and the outage constraints with $\gamma_{\mathrm{p}, 1}=-10 \mathrm{~dB}$, where the throughput is maximized over the sensing time, $R_{\mathrm{s}}\left(\tau_{\text {est }}, \tilde{\tau}_{\text {sen }}\right)$. The estimation-sensing-throughput tradeoff is utilized to determine a suitable estimation time $\tilde{\tau}_{\text {est }}$ that maximizes the throughput, $R_{\mathrm{s}}\left(\tilde{\tau}_{\text {est }}, \tilde{\tau}_{\text {sen }}\right)$.

consequence to the estimation-sensing-throughput tradeoff, we determine the suitable estimation time that yields an achievable throughput $R_{\mathrm{s}}\left(\tilde{\tau}_{\text {est }}, \tilde{\tau}_{\text {sen }}\right)$.

Besides that, we consider the variation in the achievable throughput for different values of the outage constraint, refer to Fig. 9. It is observed that for the selected choice of $\kappa$, the outage constraint is severe as compared to the average constraint, hence, results in a lower throughput. Thus, depending on the nature of policy (aggressive or conservative) followed by the regulatory bodies towards the interference at the primary system, it is possible to define $\kappa$ accordingly during the system design. Moreover, it is observed that the alternative approach proposed in Corollary 1 does not present any noticeable performance difference depicted in terms of the achievable throughput corresponding to the one characterized in the Theorems 1 and 2 .

To procure further insights, we investigate the variations of expected $\mathrm{P}_{\mathrm{d}}$ and $\mathrm{P}_{\mathrm{fa}}$ with the estimation time. From Fig. 10a, it is observed that the expected $P_{d}$ corresponding to the outage constraint is strictly above the desired level $\overline{\mathrm{P}}_{\mathrm{d}}$ for all values of estimation time, however, for lower values of estimation time, this margin reduces. This is based on the fact that lower estimation time shifts the probability mass of $\mathrm{P}_{\mathrm{d}}$, to a lower value, refer to Fig. 3a. According to Fig. 10b, the system notices a considerable improvement in $\mathrm{P}_{\mathrm{fa}}$ at small values of $\tau_{\text {est }}$, which saturates for a certain period and falls drastically beyond a certain value. To understand this, it is important to study the dynamics between the estimation and the sensing time. Low $\tau_{\text {est }}$ increases the variations in the detection probability, these variations are compensated by an increase in the suitable sensing time, and vice versa. The performance improves until a maximum $\left(\tilde{\tau}_{\text {est }}, \tilde{\tau}_{\text {sen }}\right)$ is reached, beyond this, the time resources (allocated in terms of the sensing and the estimation time) contribute more in improving the detector's performance (in terms of $P_{f a}$ as $P_{d}$ is already constrained) and less in reducing the variations due to channel (a)

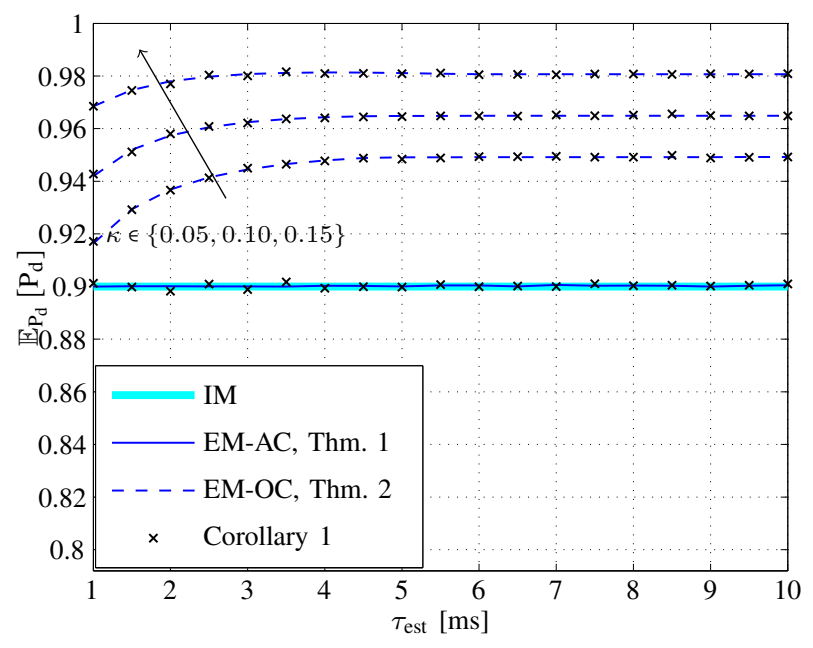

(b)

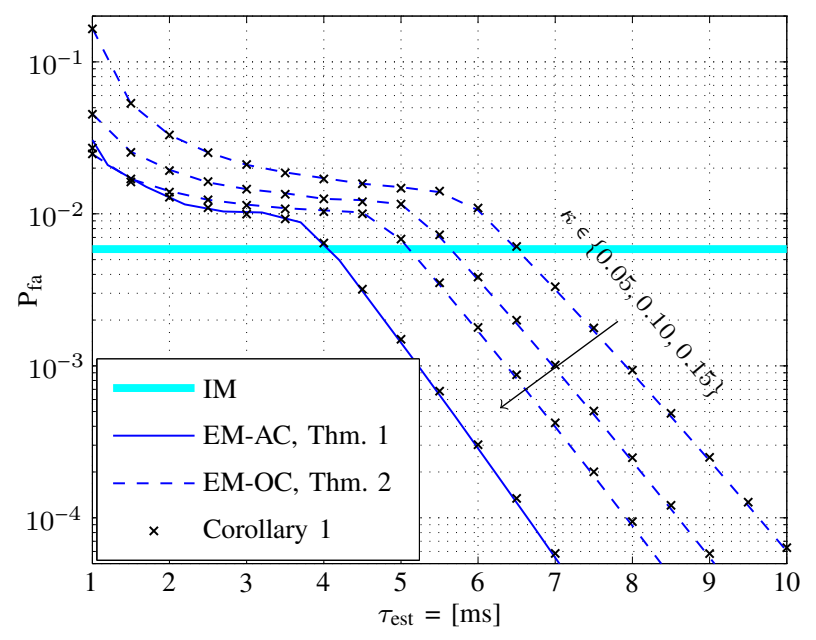

Fig. 10. Variation of $\mathbb{E}_{\mathrm{P}_{\mathrm{d}}}\left[\mathrm{P}_{\mathrm{d}}\right]$ and $\mathrm{P}_{\text {fa }}$ versus the $\tau_{\text {est }}$, where the secondary throughput is maximized over the sensing time, $R_{\mathrm{S}}\left(\tau_{\text {est }}, \tilde{\tau}_{\text {sen }}\right)$. (a) Expected $\mathrm{P}_{\mathrm{d}}$ versus $\tau_{\text {est }}$, (b) $\mathrm{P}_{\text {fa }}$ versus $\tau_{\text {est }}$.

estimation.

\section{CONCLUSION}

In this paper, we have investigated the performance of cognitive radio as an interweave system from a deployment perspective. It has been argued that the knowledge of the interacting channels is a key aspect that enables the performance characterization of the interweave system in terms of sensing-throughput tradeoff. In this regard, a novel framework that facilitates channel estimation and captures the effect of estimation in the system model has been proposed. As a major outcome of the analysis, it has been justified that the existing model, illustrating an ideal scenario, overestimates the performance of the interweave system, hence, less suitable for deployment. Moreover, it has been clearly stated that the variations induced in the system, specially in the detection probability may severely degrade the performance of the primary system. To overcome this situation, average and outage constraints as primary user constraints have been employed. As a consequence, for the proposed estimation model, novel 
expressions for sensing-throughput tradeoff based on the mentioned constraints have been established. More importantly, by analyzing the estimation-sensing-throughput tradeoff, the suitable estimation time and the suitable sensing time that maximize the secondary throughput have been determined. In our future work, we plan to extend the proposed analysis for the hybrid cognitive radio system that combines the advantages of interweave and underlay techniques.

\section{APPENDIX}

\section{A. Proof of Lemma 3}

Proof: For simplification, we break down the expression $\left(\frac{\left|\hat{h}_{\mathrm{s}}\right|^{2} P_{\mathrm{T} \mathrm{S}, \mathrm{ST}}}{\hat{P}_{\mathrm{Rx}, \mathrm{SR}}}\right)$ in (9), as $E_{1}=\left(\frac{\left|\hat{h}_{\mathrm{S}}\right|^{2} P_{\mathrm{Tx}, \mathrm{ST}}}{\sigma_{w}^{2}}\right)$ and $E_{2}=\left(\frac{\hat{P}_{\mathrm{Rx}, \mathrm{SR}}}{\sigma_{w}^{2}}\right)$, where $\mathrm{C}_{1}=\log _{2}\left(1+\frac{E_{1}}{E_{2}}\right)$. The pdf of the expression $E_{1}$ is determined in (24).

Following the characterization $\hat{P}_{\mathrm{Rx}, \mathrm{SR}}$ in (16), the pdf of $E_{2}$ is determined as

$$
\begin{aligned}
f_{\frac{\hat{P}_{\mathrm{Rx}, \mathrm{SR}}}{\sigma_{w}^{2}}} & =\frac{N_{\mathrm{p}, 2} \sigma_{w}^{2}}{P_{\mathrm{Rx}, \mathrm{SR}}} \frac{1}{2^{\frac{N_{\mathrm{p}, 2}}{2}} \Gamma\left(\frac{N_{\mathrm{p}, 2}}{2}\right)}\left(x \frac{N_{\mathrm{p}, 2} \sigma_{w}^{2}}{P_{\mathrm{Rx}, \mathrm{SR}}}\right)^{\frac{N_{\mathrm{p}, 2}}{2}-1} \times \\
& \exp \left(-x \frac{N_{\mathrm{p}, 2} \sigma_{w}^{2}}{2 P_{\mathrm{Rx}, \mathrm{SR}}}\right) .
\end{aligned}
$$

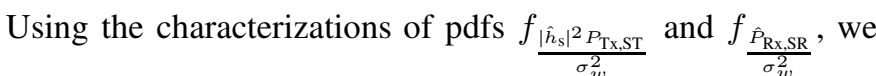
apply Mellin transform [37] to determine the pdf of $\frac{{ }^{\sigma_{w}^{2}}}{E_{2}}$ as

$$
f_{\frac{\left|\hat{h}_{\mathrm{S}}\right|^{2} P_{\mathrm{TX}, \mathrm{ST}}}{\sigma_{w}^{2}}} / \frac{\hat{P}_{\mathrm{Rx}, \mathrm{SR}}}{\sigma_{w}^{2}}(x)=\frac{x^{a_{1}-1} \Gamma\left(a_{1}+a_{2}\right)}{\Gamma\left(a_{1}\right) \Gamma\left(a_{2}\right) b_{1}^{a_{1}} b_{2}^{a_{2}}}\left(\frac{1}{b_{2}}+\frac{x}{b_{1}}\right)^{\left(a_{1}+a_{2}\right)} .
$$

Finally, substituting the expression $\frac{E_{1}}{E_{2}}$ in $\mathrm{C}_{1}$ yields (26).

\section{B. Proof of Theorems 1 and 2}

Proof: In order to solve the constrained optimization problems illustrated in Theorem 1 and Theorem 2, the following approach is considered. As a first step, an underlying constraint is employed to determine $\mu$ as a function of the $\tau_{\text {sen }}$ and $\tau_{\text {est }}$.

For the average constraint, the expression $\mathbb{E}_{\mathrm{P}_{\mathrm{d}}}\left[\mathrm{P}_{\mathrm{d}}\right]$ in (29) did not lead to a closed form expression, consequently, no analytical expression of $\mu$ is obtained. In this context, we procure $\mu$ for the average constraint numerically from (29).

Next, we determine $\mu$ based on the outage constraint. This is accomplished by combining the expression of $F_{\mathrm{P}_{\mathrm{d}}}$ in (17) with the outage constraint (31)

$$
P\left(\mathrm{P}_{\mathrm{d}} \leq \overline{\mathrm{P}}_{\mathrm{d}}\right)=F_{\mathrm{P}_{\mathrm{d}}}\left(\overline{\mathrm{P}}_{\mathrm{d}}\right) \leq \kappa .
$$

Rearranging (38) gives

$$
\mu \geq \frac{4 P_{\mathrm{Rx}, \mathrm{ST}} \Gamma^{-1}\left(1-\kappa, \frac{\tau_{\mathrm{est}} f_{\mathrm{s}}}{2}\right) \Gamma^{-1}\left(\overline{\mathrm{P}}_{\mathrm{d}}, \frac{\tau_{\mathrm{sen}} f_{\mathrm{s}}}{2}\right)}{\tau_{\mathrm{est}} \tau_{\mathrm{sen}}\left(f_{\mathrm{s}}\right)^{2}} .
$$

Clearly, the random variables $\mathrm{P}_{\mathrm{d}}\left(\hat{P}_{\mathrm{Rx}, \mathrm{ST}}\right)$, and $\mathrm{C}_{0}\left(\left|\hat{h}_{\mathrm{s}}\right|^{2}\right)$ and $\mathrm{C}_{1}\left(\left|\hat{h}_{\mathrm{s}}\right|^{2}, \hat{P}_{\mathrm{Rx}, \mathrm{SR}}\right)$ are functions of the independent random variables $\hat{P}_{\mathrm{Rx}, \mathrm{ST}}$, and $\left|\hat{h}_{\mathrm{s}}\right|^{2}$ and $\hat{P}_{\mathrm{Rx}, \mathrm{SR}}$, respectively. In this context, we apply the independence property on $\mathrm{P}_{\mathrm{d}}, \mathrm{C}_{0}$ and $\mathrm{C}_{1}$ to obtain

$$
\begin{aligned}
\mathbb{E}_{\mathrm{P}_{\mathrm{d}}, \mathrm{C}_{0}, \mathrm{C}_{1}}\left[\mathrm{C}_{0}\left(1-\mathrm{P}_{\mathrm{fa}}\right)+\mathrm{C}_{1}\left(1-\mathrm{P}_{\mathrm{d}}\right)\right] & =\mathbb{E}_{\mathrm{C}_{0}}\left[\mathrm{C}_{0}\right]\left(1-\mathrm{P}_{\mathrm{fa}}\right)+ \\
& \mathbb{E}_{\mathrm{C}_{1}}\left[\mathrm{C}_{1}\right] \mathbb{E}_{\mathrm{P}_{\mathrm{d}}}\left[\left(1-\mathrm{P}_{\mathrm{d}}\right)\right]
\end{aligned}
$$

in (28) and (30). Upon replacing the respective thresholds in $\mathrm{P}_{\mathrm{d}}$ and $\mathrm{P}_{\mathrm{fa}}$ and evaluating the expectation over $\mathrm{P}_{\mathrm{d}}, \mathrm{C}_{0}$ and $\mathrm{C}_{1}$ using the distribution functions characterized in Lemma 1, Lemma 2 and Lemma 3, we determine the expected throughput as a function of sensing and estimation time.

\section{REFERENCES}

[1] A. Kaushik, S. K. Sharma, S. Chatzinotas, B. Ottersten, and F. K. Jondral, "Sensing-Throughput Tradeoff for Cognitive Radio Systems with Unknown Received Power," in 10th International Conference on Cognitive Radio Oriented Wireless Networks and Communications (CROWNCOM), April 2015.

[2] J. Andrews, S. Buzzi, W. Choi, S. Hanly, A. Lozano, A. Soong, and J. Zhang, "What Will 5G Be?" IEEE Journal on Selected Areas in Communications, vol. 32, no. 6, pp. 1065-1082, June 2014.

[3] T. Rappaport, S. Sun, R. Mayzus, H. Zhao, Y. Azar, K. Wang, G. Wong, J. Schulz, M. Samimi, and F. Gutierrez, "Millimeter Wave Mobile Communications for 5G Cellular: It Will Work!" IEEE Access, vol. 1, pp. 335-349, 2013.

[4] A. Goldsmith, S. Jafar, I. Maric, and S. Srinivasa, "Breaking Spectrum Gridlock With Cognitive Radios: An Information Theoretic Perspective," Proceedings of the IEEE, vol. 97, no. 5, pp. 894-914, May 2009.

[5] S. Sharma, T. Bogale, S. Chatzinotas, B. Ottersten, L. Le, and X. Wang, "Cognitive Radio Techniques under Practical Imperfections: A Survey," IEEE Communications Surveys Tutorials, vol. 17, no. 4, pp. 1858-1884, Fourthquarter 2015.

[6] E. Axell, G. Leus, E. Larsson, and H. Poor, "Spectrum Sensing for Cognitive Radio : State-of-the-Art and Recent Advances," IEEE Signal Processing Magazine, vol. 29, no. 3, pp. 101-116, May 2012.

[7] H. Urkowitz, "Energy detection of unknown deterministic signals," Proceedings of the IEEE, vol. 55, no. 4, pp. 523 - 531, April 1967.

[8] V. Kostylev, "Energy detection of a signal with random amplitude," in IEEE ICC, vol. 3, 2002, pp. 1606-1610.

[9] F. Digham, M.-S. Alouini, and M. K. Simon, "On the energy detection of unknown signals over fading channels," in IEEE ICC, vol. 5, May 2003 , pp. 3575-3579.

[10] S. Herath, N. Rajatheva, and C. Tellambura, "Unified Approach for Energy Detection of Unknown Deterministic Signal in Cognitive Radio Over Fading Channels," in IEEE ICC Workshops, June 2009, pp. 1-5.

[11] A. Mariani, A. Giorgetti, and M. Chiani, "Energy detector design for cognitive radio applications," in 2010 International Waveform Diversity and Design Conference (WDD), August 2010, pp. 053-057.

[12] E. Peh and Y.-C. Liang, "Optimization for cooperative sensing in cognitive radio networks," in IEEE Wireless Communications and Networking Conference (WCNC), March 2007, pp. 27-32.

[13] Y.-C. Liang, Y. Zeng, E. Peh, and A. T. Hoang, "Sensing-Throughput Tradeoff for Cognitive Radio Networks," IEEE Transactions on Wireless Communications, vol. 7, no. 4, pp. 1326-1337, April 2008.

[14] S. Sharma, S. Chatzinotas, and B. Ottersten, "A hybrid cognitive transceiver architecture: Sensing-throughput tradeoff," in CROWNCOM, June 2014.

[15] H. Pradhan, S. Kalamkar, and A. Banerjee, "Sensing-Throughput Tradeoff in Cognitive Radio With Random Arrivals and Departures of Multiple Primary Users," IEEE Communications Letters, vol. 19, no. 3, pp. 415418, March 2015.

[16] M. Cardenas-Juarez and M. Ghogho, "Spectrum Sensing and Throughput Trade-off in Cognitive Radio under Outage Constraints over Nakagami Fading," IEEE Communications Letters, vol. 15, no. 10, pp. 11101113, October 2011.

[17] Y. Sharkasi, M. Ghogho, and D. McLernon, "Sensing-throughput tradeoff for OFDM-based cognitive radio under outage constraints," in ISWCS, August 2012, pp. 66-70.

[18] P. Stoica and O. Besson, "Training sequence design for frequency offset and frequency-selective channel estimation," IEEE Transactions on Communications, vol. 51, no. 11, pp. 1910-1917, November 2003. 
[19] W. Gifford, M. Win, and M. Chiani, "Diversity with practical channel estimation," IEEE Transactions on Wireless Communications, vol. 4, no. 4, pp. 1935-1947, July 2005.

[20] _ " "Antenna subset diversity with non-ideal channel estimation," IEEE Transactions on Wireless Communications, vol. 7, no. 5, pp. 15271539, May 2008.

[21] V. Chavali and C. da Silva, "Collaborative spectrum sensing based on a new snr estimation and energy combining method," IEEE Transactions on Vehicular Technology, vol. 60, no. 8, pp. 4024-4029, October 2011.

[22] S. Sharma, S. Chatzinotas, and B. Ottersten, "SNR Estimation for Multidimensional Cognitive Receiver under Correlated Channel/Noise," IEEE Transactions on Wireless Communications, vol. 12, no. 12, pp. 63926405, December 2013.

[23] A. Kaushik, M. Mueller, and F. K. Jondral, "Cognitive Relay: Detecting Spectrum Holes in a Dynamic Scenario," in ISWCS, April 2013, pp. $1-2$.

[24] T. Wang, Y. Chen, E. Hines, and B. Zhao, "Analysis of effect of primary user traffic on spectrum sensing performance," in Fourth International Conference on Communications and Networking in China, Aug 2009, pp. $1-5$.

[25] L. Tang, Y. Chen, E. Hines, and M.-S. Alouini, "Effect of Primary User Traffic on Sensing-Throughput Tradeoff for Cognitive Radios," IEEE Transactions on Wireless Communications, vol. 10, no. 4, pp. $1063-$ 1068, April 2011.

[26] B. Zhao, Y. Chen, C. He, and L. Jiang, "Performance analysis of spectrum sensing with multiple primary users," IEEE Transactions on Vehicular Technology, vol. 61, no. 2, pp. 914-918, February 2012.

[27] S. Kay, Fundamentals of Statistical Signal Processing: Detection theory, ser. Prentice Hall Signal Processing Series. Prentice-Hall PTR, 1998.

[28] R. Tandra and A. Sahai, "SNR Walls for Signal Detection," IEEE Journal of Selected Topics in Signal Processing, vol. 2, no. 1, pp. 4-17, Feb 2008.

[29] I. S. Gradshteyn and I. M. Ryzhik, Table of Integrals, Series, and Products, 6th ed. San Diego, CA: Academic Press., 2000.

[30] M. Gans, "The Effect of Gaussian Error in Maximal Ratio Combiners," IEEE Transactions on Communication Technology, vol. 19, no. 4, pp. 492-500, August 1971.

[31] R. Annavajjala and L. Milstein, "Performance analysis of linear diversity-combining schemes on Rayleigh fading channels with binary signaling and Gaussian weighting errors," IEEE Transactions on Wireless Communications, vol. 4, no. 5, pp. 2267-2278, Septemeber 2005.

[32] H. Suraweera, P. Smith, and M. Shafi, "Capacity limits and performance analysis of cognitive radio with imperfect channel knowledge," IEEE Transactions on Vehicular Technology, vol. 59, no. 4, pp. 1811-1822, May 2010.

[33] H. Kim, H. Wang, S. Lim, and D. Hong, "On the Impact of Outdated Channel Information on the Capacity of Secondary User in Spectrum Sharing Environments," IEEE Transactions on Wireless Communications, vol. 11, no. 1, pp. 284-295, January 2012.

[34] A. Kaushik, S. K. Sharma, S. Chatzinotas, B. Ottersten, and F. K. Jondral, "Estimation-Throughput Tradeoff for Underlay Cognitive Radio Systems," in IEEE International Conference on Communications (ICC), 2015, pp. 7701-7706.

[35] N. Cao, M. Mao, Y. Chen, and M. Long, "Analysis of collaborative spectrum sensing with binary phase shift keying signal power estimation errors," IET Science, Measurement and Technology, vol. 8, no. 6, pp. 350-358, 2014.

[36] M. Abramowitz and I. A. Stegun, Handbook of Mathematical Functions with Formulas, Graphs, and Mathematical Tables, ninth Dover printing, tenth GPO printing ed. New York: Dover, 1964

[37] F. W. J. Olver, D. W. Lozier, R. F. Boisvert, and C. W. Clark, Eds., NIST Handbook of Mathematical Functions. New York, NY: Cambridge University Press, 2010.

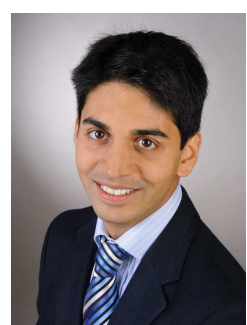

Ankit Kaushik (S'12) received the B. Tech degree in electronics and communication engineering from Guru Gobind Singh Indraprastha University, Delhi, India in 2005. He further received the dual M. Sc. degree in information and communication technology from University of Karlsruhe (now Karlsruhe Institute of Technology), Karlsruhe, Germany and Politecnico di Torino, Turin, Italy in 2007. From 2007-2012, he was with Leica Camera AG, Germany, where he worked as a Design Engineer. Since 2012, he has been working toward the Doctoral degree with the Communications Engineering Lab, Karlsruhe Institute of Technology, as a Research Associate. During the winter semester 2015/2016, he was a Visiting Researcher with the Interdisciplinary Centre for Security, Reliability and Trust (SnT), University of Luxembourg, Luxembourg. His research interests include software defined radio, cognitive radio communications and networks. He was a recipient of MERIT Scholarship for his Masters Studies within the Erasmus Mundus Scholarship Program and Subjective Winner of 5G Spectrum Challenge held at 2015 IEEE DySPAN conference.

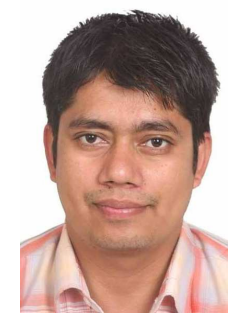

Shree Krishna Sharma (S'12-M'15) received the M.Sc. degree in information and communication engineering from the Institute of Engineering, Pulchowk, Nepal, in 2010; the M.A. degree in economics from Tribhuvan University, Nepal; the M.Res. degree in computing science from Staffordshire University, Staffordshire, U.K., in 2011; and the Ph.D. degree in Wireless Communications from University of Luxembourg, Luxembourg in 2014. Since November 2014, he has been working as a Research Associate in Interdisciplinary Centre for Security, Reliability and Trust (SnT), University of Luxembourg, Luxembourg.

In the past, Dr. Sharma was also involved with Kathmandu University, Dhulikhel, Nepal, as a Teaching Assistant, and he worked as a Part-Time Lecturer for eight engineering colleges in Nepal. He was with Nepal Telecom for more than four years as a Telecom Engineer in the field of information technology and telecommunication. He is the author of more than 50 technical papers in refereed international journals, scientific books, and conferences. He received an Indian Embassy Scholarship for his B.E. study, an Erasmus Mundus Scholarship for his M. Res. study, and an AFR Ph.D. grant from the National Research Fund (FNR) of Luxembourg. He received Best Paper Award in CROWNCOM 2015 conference held in Doha, Qatar and for his Ph.D. thesis, he received FNR award for outstanding PhD Thesis 2015 from FNR, Luxembourg. He has been involved in EU FP7 CoRaSat project, EU H2020 project SANSA, ESA project ASPIM, and Luxembourgish national projects Co2Sat, and SeMIGod. He has been serving as a reviewer for several international journals and conferences, and also as a TPC member for a number of conferences. His research interests include cognitive wireless communications, satellite communications, and signal processing techniques for $5 \mathrm{G}$ and beyond wireless. 


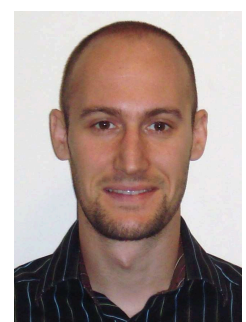

Symeon Chatzinotas (S'06-M'09-SM'13) received the M.Eng. in Telecommunications from Aristotle University of Thessaloniki, Greece and the M.Sc. and $\mathrm{Ph} . \mathrm{D}$. in Electronic Engineering from University of Surrey, UK in 2003, 2006 and 2009 respectively. He is currently a Research Scientist with the research group SIGCOM in the Interdisciplinary Centre for Security, Reliability and Trust, University of Luxembourg, managing H2020, ESA and FNR projects.

In the past, he has worked in numerous RD projects for the Institute of Informatics Telecommunications, National Center for Scientific Research Demokritos, the Institute of Telematics and Informatics, Center of Research and Technology Hellas and Mobile Communications Research Group, Center of Communication Systems Research, University of Surrey. He has authored more than 120 technical papers in refereed international journals, conferences and scientific books. His research interests are on multiuser information theory, cooperative/cognitive communications and wireless networks optimization. Dr Chatzinotas is the corecipient of the 2014 Distinguished Contributions to Satellite Communications Award, Satellite and Space Communications Technical Committee, IEEE Communications Society and CROWNCOM 2015 Best Paper Award. $\mathrm{He}$ is one of the editors of a book on "Cooperative and Cognitive Satellite Systems" published in 2015 by Elsevier and was involved in coorganizing the First International Workshop on Cognitive Radios and Networks for Spectrum Coexistence of Satellite and Terrestrial Systems (CogRaN-Sat) in conjunction with the IEEE ICC 2015, 8-12 June 2015, London, UK.

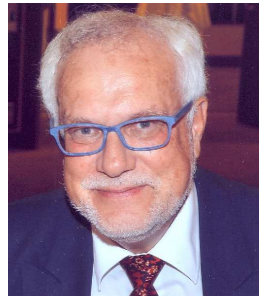

Friedrich K. Jondral (SM'94) K. Jondral received a diploma in mathematics (Dipl.-Math.) and a doctoral degree in natural sciences (Dr.rer.nat.) from the Technische Universität Braunschweig (Germany) in 1975 and 1979, respectively. During the winter semester 1977/78, he was a visiting scientist to Professor Takeyuki Hida, Ph.D., at the Department of Mathematics of Nagoya University, Nagoya (Japan). From 1979 to 1992, Dr. Jondral was an employee of AEG-Telefunken (now Airbus Defence and Space), Ulm (Germany), where he held various research, development and management positions. During this period he also lectured on applied mathematics at the Universität Ulm, where he was appointed Adjunct Professor in 1991.

In 1993 Dr. Jondral became Full Professor and Director of the Communications Engineering Lab (CEL) at the Universität Karlsruhe (TH) (Germany, now Karlsruhe Institute of Technology, KIT). Here, from 2000 to 2002, he served as the Dean of the Department of Electrical Engineering and Information Technology. During the summer semester 2005, he was a visiting faculty to Virginia Tech, Blacksburg (VA). He retired in 2015.

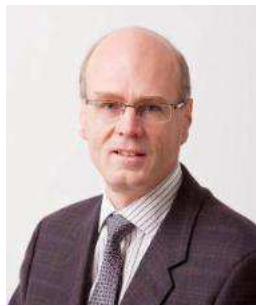

Björn Ottesten (S'87-M'89-SM'99-F'04) was born in Stockholm, Sweden, in 1961. He received the M.S. degree in electrical engineering and applied physics from Linköping University, Linköping, Sweden, in 1986 and the Ph.D. degree in electrical engineering from Stanford University, Stanford, CA, in 1989.

Dr. Ottersten has held research positions at the Department of Electrical Engineering, Linköping University, the Information Systems Laboratory, Stanford University, the Katholieke Universiteit Leuven, Leuven, and the University of Luxembourg. During 96/97, he was Director of Research at ArrayComm Inc, a start-up in San Jose, California based on Ottersten' s patented technology. He has co-authored journal papers that received the IEEE Signal Processing Society Best Paper Award in 1993, 2001, 2006, and 2013 and 3 IEEE conference papers receiving Best Paper Awards. In 1991, he was appointed Professor of Signal Processing at the Royal Institute of Technology (KTH), Stockholm. From 1992 to 2004, he was head of the department for Signals, Sensors, and Systems at KTH and from 2004 to 2008, he was dean of the School of Electrical Engineering at KTH. Currently, he is Director for the Interdisciplinary Centre for Security, Reliability and Trust at the University of Luxembourg. As Digital Champion of Luxembourg, he acts as an adviser to European Commissioner Neelie Kroes.

Dr. Ottersten has served as Associate Editor for the IEEE TRANSACTIONS ON SIGNAL PROCESSING and on the editorial board of IEEE Signal Processing Magazine. He is currently editor in chief of EURASIP Signal Processing Journal and a member of the editorial boards of EURASIP Journal of Applied Signal Processing and Foundations and Trends in Signal Processing. He is a Fellow of the IEEE and EURASIP and a member of the IEEE Signal Processing Society Board of Governors. In 2011, he received the IEEE Signal Processing Society Technical Achievement Award. He is a first recipient of the European Research Council advanced research grant. His research interests include security and trust, reliable wireless communications, and statistical signal processing. 